\title{
Perspectiva temporal: Una revisión sistematizada de su relación empírica con
} factores socioeconómicos y psicológicos

\section{Time Perspective: A systematized Review of Its Empirical Relationship With Socioeconomic and Psychological Factors}

\author{
Luque Berkowitz, Jorge Iván ${ }^{1 *}$ y Laborín Álvarez, Jesús Francisco ${ }^{1}$
}

\begin{abstract}
Resumen:
Existe un creciente interés por el constructo perspectiva temporal (PT), el cual describe la influencia de marcos temporales en la cognición y el comportamiento. Se ha planteado que la PT se relaciona con el estatus socioeconómico. Donde una orientación al futuro influye en el manejo de finanzas personales, mayores logros educativos y mejor cuidado de la salud; no obstante, participantes bajo esta orientación suelen reportar bajos niveles de felicidad y satisfacción. En contraste, la orientación al presente se vincula con ingresos bajos, menor nivel educativo y cuidado deficiente de la salud, pero mayor felicidad y satisfacción. El objetivo del artículo es mostrar evidencia científica, mediante una revisión sistematizada, acerca de la relación entre PT y factores socioeconómicos y psicológicos, durante el periodo 1999 - 2020. Con ello se busca analizar el papel que juega la PT para el funcionamiento humano. Los resultados muestran una alta producción en el número de publicaciones. Finalmente, se discuten los alcances teóricos y metodológicos.
\end{abstract}

Palabras Clave: perspectiva temporal, revisión sistematizada, factores socioeconómicos, factores psicológicos, inventario de perspectiva temporal de Zimbardo.

\begin{abstract}
:
There is a growing interest in the time perspective (TP) construct, which describes the influence of time frames on cognition and behavior. It has been suggested that TP is related to socioeconomic status. Where a future orientation influences the management of personal finances, the attainment of higher educational achievements and better health care; however, participants under this orientation tend to report low levels of happiness and satisfaction. In contrast, orientation to the present is associated with low income, lower educational level and poor health care, but greater happiness and satisfaction. The objective of the article is to show through a systematic review, scientific evidence of the relationship between TP and socioeconomic and psychological factors, during the period 19992020 , the aim is to analyze the role that TP plays on human functioning. The results show a high production in the number of publications. Finally, the explanatory and methodological scopes are discussed .
\end{abstract}

Keywords: time perspective, systematic review, socioeconomic factors, psychological factors, Zimbardo Time Perspective Inventory.

${ }^{1}$ Centro de Investigación en Alimentación y Desarrollo A.C., Hermosillo, México.

*Correspondencia: ivan.luque@estudiantes.ciad.mx 
La perspectiva del tiempo, una dimensión fundamental en la construcción del tiempo psicológico, surge de los procesos cognitivos que dividen la experiencia humana en marcos (Aquino, et al., 2017; Frank1, 1988).

El tiempo subjetivo ha sido objeto de estudio para la psicología desde sus inicios como disciplina científica (Worrel, 2021). Parte de esta tradición, ha girado en torno a cómo influyen los pensamientos sobre el pasado, el presente y el futuro en el comportamiento y la cognición de individuos y sociedades.

El concepto de perspectiva temporal (PT) refiere un constructo cognitivo y motivacional. Se considera cognitivo ya que se fundamenta en los pensamientos y procesos mentales de las personas. De igual forma, es motivacional porque dichos pensamientos sobre el tiempo llevan a las personas a tomar decisiones y a ejercer comportamientos específicos (Mello \& Worrell, 2015).

El concepto fue planteado por Lewin (1942), quien la define como la visión que un individuo tiene de su pasado y su futuro psicológico en un momento presente.

Tradicionalmente, el estudio de la PT se ha había desarrollado por medio de instrumentos centrados solamente en el futuro (Nuttin \& Lens, 1984; Stratham, et al., 1994; Seginer, 2017). Sin embargo, estudios más recientes consideran también el pasado y el presente (Stolarski, et al., 2015).

Un enfoque de creciente interés, es el constructo de perspectiva temporal (PT) desarrollado por Zimbardo y Boyd (1999), quienes la definen como un proceso generalmente inconsciente, por medio del cual se organizan experiencias y expectativas en marcos temporales, que ayudan a darle sentido y coherencia a la vida. La PT es, por un lado, un proceso cognitivo constante en el cual la atención se centra en marcos temporales; y por otro lado, cada individuo puede desarrollar una tenden- cia disposicional de uso y abuso de algún marco temporal específico, generando así una especie de sesgo, que influye en la toma de decisiones, el comportamiento y en la manera de ver el mundo (Laghi, et al., 2009).

En el enfoque de Zimbardo y Boyd (1999), la PT se compone de cinco factores o marcos temporales: el pasado positivo (PP), que hace referencia a un sentimiento de apego y calidez hacia el pasado; el pasado negativo $(\mathrm{PN})$, que representa una visión aversiva del pasado, relacionada a vivencias negativas; el presente hedonista $(\mathrm{PH})$, que alude a una fuerte orientación hacia el placer inmediato y a una baja consideración de consecuencias futuras (Stratham, et al., 1994); el presente fatalista (PF), que implica una actitud de desesperanza e impotencia ante las condiciones de la vida; así como de vivir sometido a un destino del cual no se tiene control alguno; y finalmente, la perspectiva temporal del futuro (PTF), la cual representa un comportamiento diligente orientado al logro, las personas bajo esta perspectiva se centran en recompensas, logros y presentan una alta consideración de consecuencias futuras (Strathman, et al., 1994), por lo que representa el marco temporal más adaptativo (Mello, 2019; Zimbardo \& Boyd, 2009).

Cada individuo acude a un marco temporal específico de manera habitual, esto depende de diversos factores entre los que se encuentran la edad, el género, el nivel educativo, la familia, el contexto social y el nivel socioeconómico, así como la maduración cognitiva (International Time Perspective Network, 2020). La orientación temporal, hace referencia al énfasis que uno pone, ya sea en el pasado, presente o futuro. Zimbardo y Boyd (1999), plantean la existencia de un perfil temporal óptimo, llamado perspectiva temporal balanceada (PTB). En la cual deben predominar las perspectivas positivas (PP, PTF y 
$\mathrm{PH})$ y verse reducidas las negativas (PN y $\mathrm{PF})$.

La mayoría de estudios se han enfocado en buscar relaciones entre orientaciones al futuro, o el presente, con otros constructos psicológicos, así como en analizar los efectos de tales perspectivas en el comportamiento (Keough, et al., 1999).

Desde los primeros estudios en este campo, la PT se ha postulado como un fenómeno profundamente arraigado y moldeado por las condiciones de vida (Fieulaine \& Apostolidis, 2015). Incluso se ha planteado que cada clase social se puede caracterizar por una PT dominante (Eppel, et al., 1999; Fieulaine \& Apostolidis, 2015; Trommsdorff, 1983), siendo los más necesitados los que en menor medida se orientan hacia el futuro, y en mayor medida hacia el presente. Sin embargo, enfoques más recientes ven esta relación a la inversa, donde la PT influye en el nivel socioeconómico que puede alcanzar una persona. Esto se debe a que cada PT promueve estilos cognitivoconductuales que ayudan o afectan en el desarrollo de capacidades para la movilidad social.

Por lo tanto, la PT depende de las situaciones habituales que le toca vivir a una persona o grupo, y en paralelo, el significado psicológico de esas situaciones, así como sus consecuencias, se establecen como resultado de la PT adoptada. Por lo tanto, se trata de una relación circular de interdependencia entre el individuo y el ambiente. La mayoría de los estudios en la actualidad parten del supuesto, de que es la PT la que provoca comportamientos y toma de decisiones que explican el nivel socioeconómico (NSE) (Kooij, et al., 2018; McKay, et al., 2018). No obstante, algunos estudios plantean lo opuesto (Eppel, et al., 1999; Fieulaine \& Apostolidis, 2015).

En general, las evidencias indican que orientarse hacia el futuro se relaciona con lo- gros positivos en educación (GonzalezLomelí, et al., 2006), cuidado de la salud (Hall, et al., 2015) y finanzas personales (Gore, 2018; Zimbardo, et al., 2017). Por ello la PTF suele asociarse a un nivel socioeconómico más alto. La explicación recae en que tener una visión positiva y clara del futuro, favorece un estado mental que promueve la agenciación humana y conduce a una mayor capacidad de logro (Bandura, 1997). La orientación hacia el futuro también permite planear mejor el alcance de metas, resistir tentaciones y guiarse con base en un futuro deseado (Nuttin \& Lens, 1984).

En contraste, las orientaciones hacia el presente (PH-PF) se relacionan con dificultades de desempeño en educación, cuidado de la salud y finanzas, ya que merman la extensión del horizonte temporal donde se ubica el futuro, incrementando la importancia de aquello que provoca disfrute y placer inmediato, o reduciendo la motivación. Al haber incertidumbre sobre como será el futuro, los recursos cognitivos y el comportamiento se ubican en el presente, lo que a la larga, puede tener como resultado un nivel socioeconómico bajo (Stolarski, et al., 2015).

Al respecto, la teoría de la PT plantea que personas orientadas al presente tienden a ser impacientes, poco organizados y prefieren las gratificaciones inmediatas; es por ello que suelen tener un bajo desempeño en educación, salud y manejo del dinero (Haushofer \& Fehr, 2014; Guthrie, et al., 2009; Kooij, et al., 2018; Zimbardo \& Boyd, 1999; Zimbardo, et al., 2017).

Considerando que mantener una PTF ha mostrado ser lo más óptimo para el desarrollo de una mejor posición social y económica, algunos autores han abogado por enfocar los pensamientos, actitudes y comportamientos lo más posible en el futuro. Sin embargo, Boniwell y Zimbardo (2004), advierten que una 
excesiva orientación de este tipo afecta la calidad de vida (CV), producto de un obsesivo interés en metas altamente demandantes, que pueden llegar a mermar las relaciones sociales, promover adicción al trabajo (workaholism), pobreza de tiempo, y pueden llegar a reducir el sentido de pertenencia a la comunidad, la cultura y la familia.

Por otro lado, una orientación hacia el presente en términos de placer $(\mathrm{PH})$, favorece el bienestar subjetivo, puesto que la actitud de esta perspectiva es "vivir el momento" sin preocuparse por las consecuencias futuras. Quienes se mantienen en el presente, tienden a ser sociables, alegres a la vez que impulsivos (Zimbardo \& Boyd, 1999). No obstante, lo recomendable es hacer un uso funcional de estas perspectivas. La asertividad de conducirse bajo esta visión en ciertas ocasiones depende de la ocasión misma. Tomar esta perspectiva también es una manera de disfrutar la vida, no obstante, las evidencias demuestran que adoptar ese estilo como un rasgo estable, no es recomendable para el éxito educativo (Husman, et al., 2015), o para el cuidado de la salud (Guthrie, et al., 2009).

Con base en lo anterior, destaca el importante papel que juega la PT en la vida de los seres humanos, ya que no solo influye en el despliegue de capacidades individuales que podrían permitir el alcance de estándares sociales y metas personales, sino además, se arraiga en lo más profundo de la subjetividad como un proceso que subyace a la propia conciencia (Bandura, 1999; Zimbardo \& Boyd, 1999).

No obstante, la adopción de la perspectiva más positiva en términos socioeconómicos (PTF) puede resultar inconveniente en términos psicológicos; y este efecto se produce a la inversa cuando se trata de una perspectiva $\mathrm{PH}$, al resultar más positiva en términos de felicidad y satisfacción, pero negativa para el cuidado de la salud, el desempeño escolar y el manejo de las finanzas y el ingreso. Además, existe poco consenso sobre cómo se relacionan perspectivas orientadas al pasado, con aspectos psicológicos y socioeconómicos.

En resumen, la PT ha mostrado ser un robusto predictor de aspectos relacionados al desarrollo humano y la calidad de vida, sin embargo, falta clarificar el mecanismo por el cual actúa. Por ello cabe preguntarse: ¿cómo se relacionan específicamente los diferentes marcos temporales (o factores) de la PT con el estatus socioeconómico y el bienestar subjetivo?. Para responder a esta cuestión, se llevó a cabo una revisión sistematizada de literatura donde se reportan relaciones entre PT y factores socioeconómicos y psicológicos. Específicamente felicidad, satisfacción, afecto, autoestima, depresión y ansiedad en el caso de estas últimas, e ingreso, educación y salud para las anteriores. El objetivo del presente artículo, es mostrar evidencia científica, mediante una revisión sistematizada, acerca de la relación entre PT y factores socioeconómicos y psicológicos, durante el periodo 1999 2020, con ello, se busca aportar evidencias sobre el papel que juega la PT para el funcionamiento humano.

\section{Método}

La investigación consistió en una revisión sistematizada bajo los estándares de la metodología SALSA (Search, Appraisal, Synthesis and Analysis), siguiendo las recomendaciones de Codina (2018). Esta metodología contiene cuatro fases: de búsqueda, evaluación, síntesis y análisis. Aunque de forma general, las revisiones sistematizadas de las Humanidades y Ciencias Sociales, se basan en las revisiones sistemáticas de las ciencias de la salud, se diferencian de estas últimas en que no se centran únicamente en estudios cuantitativos, ni se sintetizan los resultados con técnicas esta- 
dísticas como las de los metaanálisis. La revisión sistematizada es más flexible en su análisis y síntesis, ya que se lleva a cabo en función de las características y objetivos de cada revisión. Además, gira en torno al análisis y exploración de un área de conocimiento particular, con el fin de identificar tendencias y áreas de oportunidad (Codina, 2018).

La revisión se llevó a cabo en marzo de 2020. Se acudió a las bases de datos Scopus, Wiley Online y Springer, utilizando los términos de búsqueda "Perspectiva Temporal" y "Time Perspective", por separado. Se entrecomillaron los términos de búsqueda con el fin de obtener resultados lo más exactos posibles. Se estableció un periodo de búsqueda desde 1999, año de la primera publicación de Zimbardo y Boyd sobre PT, hasta 2020. Se refinaron los valores de búsqueda para limitar los resultados a artículos científicos del área de Ciencias Sociales y Psicología, que además estuvieran disponibles para libre acceso. Para asegurar la calidad de los resultados, se consideraron artículos empíricos, teóricos y revisiones bibliográficas que aparecieran en revistas indizadas, en español e inglés.

Posteriormente, el criterio de elegibilidad se basó en el título y/o resúmenes de cada texto, de manera que éstos reportaran relaciones entre PT y variables de dimensión socioeconómica y/o psicológica, y que su medida incluyera el futuro como dimensión temporal. Para la ubicación de estudios socioeconómicos en relación con la $\mathrm{PT}$, se seleccionaron los que en cuyo título o resumen incluyeran variables relativas a educación, salud e ingreso/finanzas, o nivel socioeconómico en general, además de la PT.

Para el caso de variables psicológicas, se eligieron las variables felicidad, afecto, satisfacción con la vida, autoestima, depresión y ansiedad. En la figura 1 se muestra el procedimiento de selección y los resultados finales.

Figura 1 Diagrama de flujo de procedimiento de selección de estudios

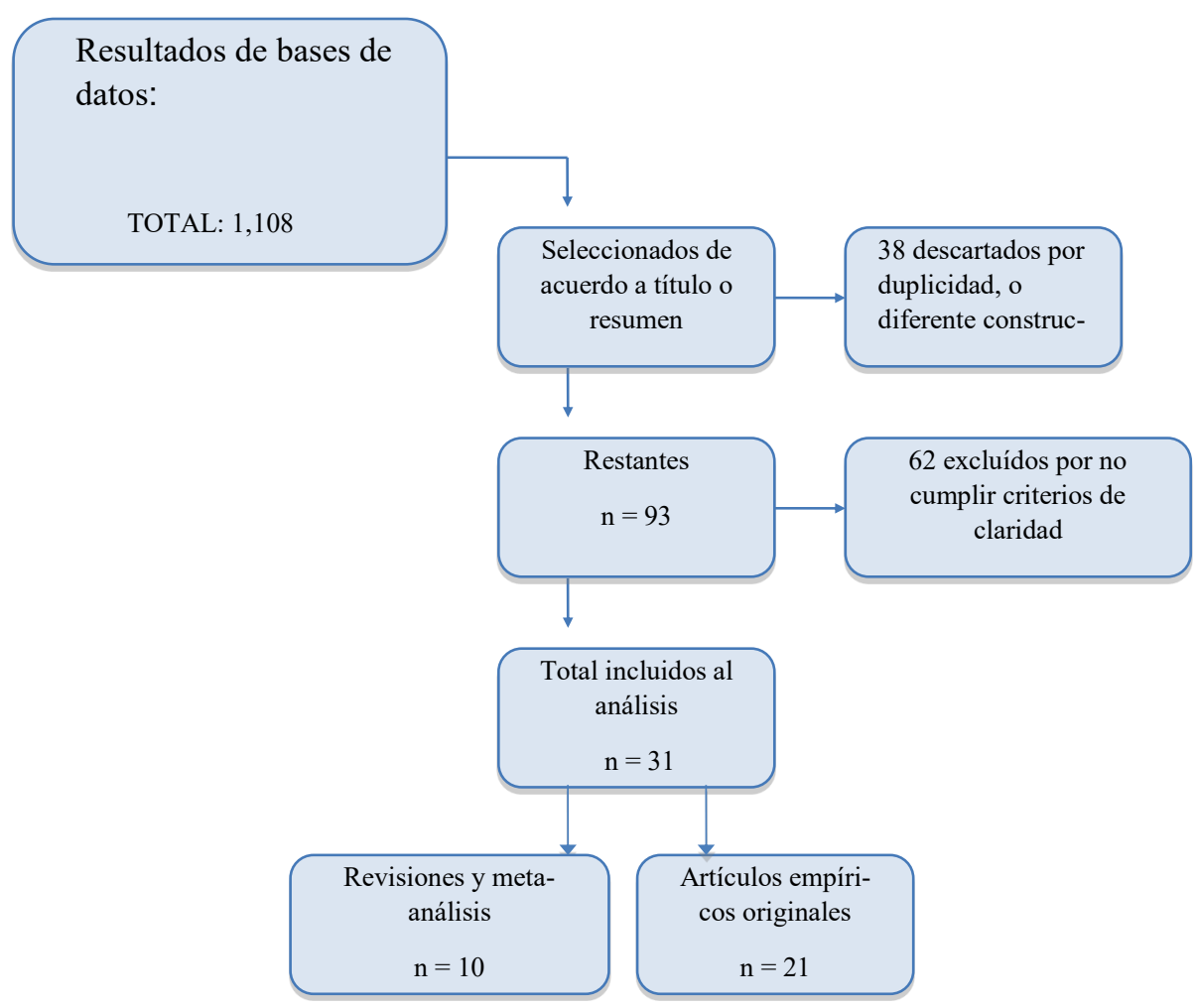


Posteriormente, se extrajeron los datos referentes a las variables utilizadas para cada investigación, así como su diseño metodológico, objeto de estudio, resultados y aportaciones principales. La codificación de estudios fue llevada a cabo por el primer autor del presente artículo. La fase final consistió en la síntesis narrativa, de forma que se pudieron identificar patrones y tendencias en los resultados, los cuales se discuten al final del artículo.

\section{Resultados}

Inicialmente, se obtuvieron 1,108 resultados de las tres bases de datos en conjunto. La gran cantidad de textos obtenidos da cuenta del interés que ha despertado el constructo en las últimas dos décadas. Al respecto, Worrell (2021), reportó haber encontrado más de 9,000 citaciones en una revisión de literatura sobre PT, con un periodo de 1950 al 2021. Lo que indica que no se trata de un constructo nuevo, y que el interés de los investigadores por el tiempo psicológico ha permanecido ya por varias décadas.

Una vez obtenidos los resultados, se revisaron el título y/o resúmenes de cada uno, con el fin de ubicar los textos que reportaran variables socioeconómicas y psicológicas en relación a la PT.

Para el caso de educación, se tomaron estudios que midieron las variables años de escolaridad, calificaciones, desempeño estudiantil, participación en el aula y persistencia académica. Para salud, se tomaron variables relativas a los hábitos alimenticios, la actividad física, la higiene, las prácticas sexuales, el apego a tratamientos médicos y el peso. Finalmente, se consideraron también el manejo de finanzas personales y el ingreso.

Por el lado de las variables psicológicas se seleccionaron felicidad, afecto, satisfacción con la vida, autoestima, depresión y ansiedad.
Una vez revisados todos los resultados. Se seleccionaron 131 textos potenciales, en cuyos títulos o resúmenes se incluía alguna o varias de las variables objetivo. Posteriormente, se ubicaron 38 duplicados, por lo que fueron excluidos; de los 93 restantes, 62 fueron excluidos tras analizar el cuerpo del artículo, ya fuera que no incluían las variables objetivo o no quedaba clara su metodología. Se seleccionaron al final 21 artículos empíricos, 8 revisiones bibliográficas y 2 meta-análisis, haciendo un total de 31 textos para análisis y síntesis.

Los resultados se muestran en la tabla 1 por orden alfabético. La columna de variables relacionadas con PT muestra las medidas utilizadas. Cabe mencionar que solo se tomaron datos referentes a las variables de interés, ya que en algunos estudios también se analizaron otras variables, pero no fueron incluidas en esta revisión. La columna de correlaciones/ efectos muestra los resultados por variable, y entre paréntesis se muestra el factor o marco temporal de referencia. En algunos casos, con el fin de optimizar espacio, se incluyeron sólo los valores más altos y más bajos de correlación; en otros se presentan las puntuaciones en cada una de las cinco categorías temporales; esto también depende de los resultados presentados en cada estudio. Se añadieron los valores de regresión en la misma columna, siempre y cuando estos fueran reportados en el artículo original de forma clara. Para el caso de las revisiones bibliográficas de tipo cualitativo, se incluyó un resumen de las principales conclusiones, haciendo énfasis en la categoría temporal que más destacó dentro del estudio citado.

Se observan en las bases de datos mayores contribuciones de Estados Unidos, Reino Unido y Países Bajos. En Latinoamérica, Argentina, Brasil y México son los países con más aportaciones. 
Tabla 1

Análisis de resultados de la revisión sistematizada sobre la relación de la PT con factores socioeconómicos y psicológicos (31 textos).

\begin{tabular}{|c|c|c|c|c|}
\hline Referencia & $\begin{array}{l}\text { Variables estudia- } \\
\text { das con relación a } \\
\text { la PT (medidas) }\end{array}$ & $\begin{array}{l}\text { Participantes, país de } \\
\text { origen, diseño }\end{array}$ & \multicolumn{2}{|c|}{ Correlaciones/efectos } \\
\hline $\begin{array}{l}\text { Anagnostopo- } \\
\text { ulos \& Griva } \\
(2012)\end{array}$ & $\begin{array}{l}\text {-Ansiedad (STAI) } \\
\text {-Auto-estima (EAR) } \\
\text {-Depresión (CES-D) }\end{array}$ & $\begin{array}{l}\text { Dos grupos de estudian- } \\
\text { tes } \\
\text { en Grecia } \\
\text { Ntotal }(1,2)=457 \\
\mathrm{n}_{1}=337 \\
226 \text { mujeres } \\
(\mathrm{DE}) \text { edad }=20.8(5.0) \\
\mathrm{n} 2=120 \\
71 \text { mujeres } \\
\mathrm{M}(\mathrm{DE}) \text { edad }=28.8(9.3)\end{array}$ & $\begin{array}{l}\text { Correlaciones positivas } \\
\text { Ansiedad-PN: } \mathrm{r}=.77^{* *} \\
\text { Depresión-PF } \mathrm{r}=.62^{* *} \\
\text { Autoestima-PP } \mathrm{r}=.19^{*}\end{array}$ & $\begin{array}{l}\text { Correlaciones negativas } \\
\text { Ansiedad-PP } \mathrm{r}=-.21^{*} \\
\text { Depresión-PP } \mathrm{r}=-.18^{*} \\
\text { Autoestima-PN } \mathrm{r}=-.57^{* *}\end{array}$ \\
\hline $\begin{array}{l}\text { Andre, van } \\
\text { Vianen, } \\
\text { Peetsma, \& } \\
\text { Oort } \\
(2018)\end{array}$ & $\begin{array}{l}\text {-Educación } \\
\text {-Trabajo } \\
\text {-Salud }\end{array}$ & $\begin{array}{l}\text { Meta-análisis } \\
\mathrm{K}=77 \\
\mathrm{~N}=31,558 \\
\text { Educación } \mathrm{k}=28 \\
\text { Trabajo } \mathrm{k}=17 \\
\text { Salud } \mathrm{k}=32\end{array}$ & $\begin{array}{l}\mathrm{r}=.24,95 \% \text { CI }[.20, .28], \quad \mathrm{p}< \\
\mathrm{Trab} \\
\mathrm{r}=.24,95 \% \text { CI }[.17, .31], \\
\mathrm{p} \\
\mathrm{Sal} \\
\mathrm{Sal} \\
\mathrm{p}\end{array}$ & $\begin{array}{l}\text { ción } \\
.0001(\mathrm{PTF}) \\
\text { ajo } \\
.0001(\mathrm{PTF}) \\
\text { ud } \\
.0001(\mathrm{PTF})\end{array}$ \\
\hline $\begin{array}{l}\text { Baird, Webb, } \\
\text { Martin, \& } \\
\text { Sirois (2018) }\end{array}$ & $\begin{array}{l}\text {-Afecto asociado al } \\
\text { monitoreo de glucosa } \\
\text { (AAMG) } \\
\text {-Actitudes hacia el } \\
\text { monitoreo de glucosa } \\
\text { (GME-Q) } \\
\text {-Frecuencia del moni- } \\
\text { toreo de glucosa } \\
\text { (FMG) }\end{array}$ & $\begin{array}{l}\text { Adultos con Diabetes } \\
\text { tipo } 1 \text { en Inglaterra } \\
\mathrm{n}=1,437 \\
704 \text { mujeres } \\
\text { M(DE)edad }=49.9(17.1)\end{array}$ & $\begin{array}{l}\mathrm{r}=.26, \mathrm{p}<.01(\mathrm{DPTB}) \\
\mathrm{r}=.18, \mathrm{p}<.05(\mathrm{DPTB}) \\
\mathrm{r}=.14, \mathrm{p}=.066(\mathrm{DPTB})\end{array}$ & $\begin{array}{l}\text { MG } \\
\text { G-Q }\end{array}$ \\
\hline $\begin{array}{l}\text { Boniwell, } \\
\text { Osin, Linley, } \\
\text { \& Ivanchenko } \\
(2010)\end{array}$ & $\begin{array}{l}\text {-Afecto positivo y } \\
\text { negativo (PANAS) } \\
\text {-Satisfacción tem- } \\
\text { poral c/vida } \\
\text { (TSWLS) } \\
\text {-Satisfacción c/la } \\
\text { vida (SWLS) } \\
\text { - Felicidad }\end{array}$ & $\begin{array}{l}\text { Dos estudios con estu- } \\
\text { diantes de Rusia y Reino } \\
\text { Unido } \\
\# 1 \text { R.U. } \\
\text { n1 }=179 \\
144 \text { mujeres } \\
\text { Medad }=24 \\
\text { DE }=\text { no reportada } \\
\# 2 \text { RUSIA } \\
\text { n2 }=289 \\
157 \text { mujeres } \\
\text { Medad }=22\end{array}$ & $\begin{array}{l}\text { Correlaciones positivas } \\
\text { TSWLS }=\mathrm{r}=.52 * * *(\mathrm{PP}) \\
\text { Afecto }+=\mathrm{r}=.34 * * *(\mathrm{PH}) \\
\text { Afecto - }=\mathrm{r}=.46 * * *(\mathrm{PN})\end{array}$ & $\begin{array}{l}\text { Correlaciones negativas } \\
\text { TSWLS }=-.62 * * *(\mathrm{PN}) \\
\text { Afecto }+=-.16^{*}(\mathrm{PF}) \\
\text { Afecto }-=-.28 * * *(\mathrm{PP})\end{array}$ \\
\hline
\end{tabular}


Tabla 1

Análisis de resultados de la revisión sistematizada sobre la relación de la PT con factores socioeconómicos y psicológicos (3 1 textos).

(Continuación)

$\begin{array}{lll}\text { Chen, Liu, Cui, } & \text {-Variables demo- } & \text { Estudiantes y habitan- } \\ \text { Chen, \& Wang } & \text { gráficas: } & \text { tes de tres ciudades en } \\ \text { (2016) } & \text {-edad } & \text { China } \\ & \text {-años de educa- } & \mathrm{n}=1,901 \\ & \text { ción } & 5 \text { grupos divididos por } \\ & \text {-Percepción de la } & \text { edad. } \\ & \text { situación econó- } & \text { (de } 9 \text { a } 65 \text { años). } \\ & \text { mica } & \text { No se reportan M(DE) } \\ & \text { (PSE) } & \text { de las edades de los } \\ & & \text { participantes }\end{array}$

Chen, Liu, Cui, Chen, Wang, Zhang, ... Chan (SWLS) (2016)

(SWLS)
-Edad

Cunningham, Zhang, \& Howell (2015)

Desmyter, \& De Raedt (2012)

Drake, Duncan, Sutherland, Abernethy, \& Henry, (2008)

Epel, Bandura, \& Zimbardo (1999)

vida

(SWLS)

trica

(GDS)
-Satisfacción c/

vida

-Satisfacción c/

vida

-Afecto positivo y negativo

-Felicidad
Estudiantes y habitantes de tres ciudades en China

$\mathrm{n}=1,598$

853 mujeres

$\mathrm{M}(\mathrm{DE}) \mathrm{edad}=34.3(18.4)$

Revisión bibliográfica donde se reportan correlaciones de varios estudios
(Puntuación media más alta)

Edad

Niños $=15.6(2.6), 95 \%$ CI $[15.2,15.9](P P)$

Adolescentes $=15.4(2.8), 95 \%$, CI $[15.2,15.7](\mathrm{PP})$

Jóvenes adultos $=14.5(2.27), 95 \%$, CI $[14.3,14.7]$ (PP)

Adulto medio $=13.6(2.4), 95 \%$, CI $[13.4,13.8](\mathrm{PTF})$

Adulto mayor $=12.6(2.2),. 95 \%$, CI $[12.3,13.0](\mathrm{PP})$

$\begin{array}{lc}\text { Educación } \\ \begin{array}{l}\text { Correlaciones positivas } \\ \mathrm{r}=.16, \mathrm{p}<.001(\mathrm{PH})\end{array} & \mathrm{r}=-.08, \mathrm{p}<.001(\mathrm{PN}) \\ \mathrm{r}=.05, \mathrm{p}<.027(\mathrm{PP}) & \\ & \\ & \text { PSE } \\ \text { Correlaciones positivas } & \text { Correlaciones negativas } \\ \mathrm{r}=.10, \mathrm{p}<.001(\mathrm{PP}) & \mathrm{r}=-.07, \mathrm{p}<.01(\mathrm{PN}) \\ & \mathrm{r}=-.10, \mathrm{p}<.001(\mathrm{PF})\end{array}$

Correlaciones positivas $\mathrm{r}=.06, \mathrm{p}<0.05(\mathrm{PF})$

Correlaciones positivas $\mathrm{r}=.179, \mathrm{p}<.01(\mathrm{PF})$

\section{SWLS}

Correlaciones negativas $\mathrm{r}=-.07, \mathrm{p}<.01(\mathrm{PF})$

Edad

Afecto positivo r's entre -.21 y -.11(PN) r's entre .24 y $.27(\mathrm{PH})$

\section{Felicidad}

r's entre .15 y .44 (PP)

r's entre .09 y $.23(\mathrm{PH})$

r's entre -.18 y -.23 (PF)

-Afecto positivo $\mathrm{y}$ negativo

(PANAS)

-Depresión geriá-

$$
\begin{array}{r}
\beta=.25^{*}, \mathrm{t}=2.72, \mathrm{r}^{2}=.37(\mathrm{PP}) \\
\beta=-.45^{*}, \mathrm{t}=5.39, \mathrm{r}^{2}=.37(\mathrm{PN}) \\
\text { PANAS }
\end{array}
$$

Afecto negativo r's entre .38 y.56 (PN) 's entre .24 y -.12 (PTF)

Satisfacción c/la vida

r's entre -.42 y -.59 (PN)

's entre .09 y $.23(\mathrm{PH})$

's entre -.16 y -.39 (PF)
Personas de la tercera edad en Bélgica

$\mathrm{n}=149$

86 mujeres

$\mathrm{M}(\mathrm{DE}) \mathrm{edad}=75.7(7)$

Afecto positivo: $\beta=.34^{*}, \mathrm{t}=3.61, \mathrm{r}^{2}=.15(\mathrm{PH})$ Afecto positivo: $\beta=.32 *, \mathrm{t}=3.17, \mathrm{r}^{2}=.15(\mathrm{PTF})$

Afecto positivo: $\beta=-.27^{*}, \mathrm{t}=5.28, \mathrm{r}^{2}=.15(\mathrm{PN})$

Afecto negativo: $\beta=.31^{*}, \mathrm{t}=3.23, \mathrm{r}^{2}=.15(\mathrm{PN})$

\section{GDS}

$\beta=.43 *, \mathrm{r}^{2}=.38(\mathrm{PN})$

$\beta=-.20, \mathrm{r}^{2}=.38(\mathrm{PH})$

Estudiantes en Escocia
(1)

$\mathrm{r}=.15, \mathrm{p}<.01(\mathrm{PH})$

$\mathrm{r}=.21, \mathrm{p}<.001(\mathrm{PP})$

$\mathrm{r}=-.41, \mathrm{p}<.001(\mathrm{PN})$

SHS

Empleo

$\mathrm{r}=.22, \mathrm{p}<.05(\mathrm{PTF})$

$\mathrm{r}=-.20, \mathrm{p}<.05(\mathrm{PH})$ $\mathrm{n}=260$
90 mujeres entre 16 y 83 años, media de edad no re- portada
Indigentes en EUA
$\mathrm{n}=82$
no se reportan medias de edad
-Duración en últi- mo o actual em- pleo -Nivel educativo
(SHS) 
Tabla 1

Análisis de resultados de la revisión sistematizada sobre la relación de la PT con factores socioeconómicos y psicológicos (31 textos). (Continuación)

\begin{tabular}{|c|c|c|c|}
\hline $\begin{array}{l}\text { Fieulaine } \\
(2017)\end{array}$ & $\begin{array}{l}\text {-Consumo de sus- } \\
\text { tancias }\end{array}$ & $\begin{array}{l}\text { Revisión bibliográfica } \\
\text { con contribuciones } \\
\text { teóricas }\end{array}$ & $\begin{array}{l}\text { Conclusión general: El impacto de la PT en el abuso de sustan } \\
\text { es mediado por otras variables como el auto-control y la perc } \\
\text { ción del riesgo. No obstante, la orientación hacia el presente } \\
\text { un factor de la adicción y el uso de sustancias. }\end{array}$ \\
\hline $\begin{array}{l}\text { Fieulaine \& } \\
\text { Apostolidis } \\
(2015)\end{array}$ & $\begin{array}{l}\text {-Ingreso } \\
\text {-Nivel educativo } \\
\text {-Exclusión social } \\
\text {-Salud Mental }\end{array}$ & $\begin{array}{l}\text { Revisión bibliográfica } \\
\text { con contribuciones } \\
\text { teóricas }\end{array}$ & $\begin{array}{l}\text { Cuando hay escasez de recursos suele haber mayor inclinaci } \\
\text { hacia el presente, producto de procesos de socialización y } \\
\text { características de los eventos habituales a los que se enfrer } \\
\text { cada estrato social. }\end{array}$ \\
\hline
\end{tabular}

GonzalezLomelí, Maytorena, Cárdenas, \& Tapia (2017)

GonzálezLomelí, Maytorena, Lohr \& Carreño 2006

Gore (2018)

Güel, Orchard, Yopo, \& Jiménez (2015)

\section{-Nacionalidad}

-Desempeño académico (DA)

-Ingreso (monto y estabilidad del mismo durante un mes)

-Satisfacción c/ vida (WVS) -Afecto positivo y negativo

(PANAS)

-Depresión (DSS)
Estudiantes universitarios de Colombia y México

$\mathrm{N}=1,265$

n-- $1=770$ colombianos

$50 \%$ mujeres

$\mathrm{n}_{2}=495=$ mexicanos

$53 \%$ mujeres

*edad no reportada

Estudiantes de tres carreras en México $\mathrm{n}=219$

Comunicación: $\mathrm{n}=68$

Psicología: $\mathrm{n}=94$

Trabajo Social: $\mathrm{n}=57$

Dos estudios, con estudiantes y adultos en

EUA

$\mathrm{n}-\mathrm{-}_{1}=300$

M(DE)edad $=20.7(5.7)$

231 mujeres

$\mathrm{n}_{2}=167 \mathrm{M}(\mathrm{DE})$

edad $=36.6(11.3)$

85 mujeres

Población adulta del medio rural y urbano en Chile

$\mathrm{n}=2,535$

(comparación de medias)

México Colombia $\mathrm{T} \quad$ sig. $\quad \underset{\text { (factor) }}{\mathrm{PT}}$

$\mathrm{M}=2.72, \quad \mathrm{M}=2.72 \quad 8.358 \quad .045 \quad \mathrm{PN}$

$\mathrm{M}=3.62, \quad \mathrm{M}=3.63, \quad 0.315 \quad .164 \quad \mathrm{PP}$

$\mathrm{M}=2.86, \quad \mathrm{M}=3.03, \quad 3.965 \quad .000 \quad \mathrm{PH}$

$\mathrm{M}=2.10, \quad \mathrm{M}=2.45, \quad 8.053 \quad .312 \quad \mathrm{PF}$

$\mathrm{M}=3.89, \quad \mathrm{M}=3.51, \quad-10.048 \quad .138 \quad \mathrm{PTF}$

DA

-Trabajo social presenta mayor tendencia a PTF (100\%) y es la que presenta el DA más alto (96.4)

-Comunicación presenta mayor tendencia a $\mathrm{PH}(71.7 \%)$ y es la que presenta el DA más bajo (62.8)

Estudio 1

(Variable: Ingreso)

Correlaciones positivas Correlaciones negativas

$\mathrm{r}=.14^{*}, \mathrm{p}<.01(\mathrm{PTF}) \quad \mathrm{r}=-.25^{*}, \mathrm{p}<.01(\mathrm{PH})$

Estudio 2

(variable: Ingreso)

Correlaciones positivas Correlaciones negativas

$\mathrm{r}=.03, \mathrm{p}<.005(\mathrm{PTF}) \quad \mathrm{r}=-.09, \mathrm{p}<.005(\mathrm{PF})$

Cluster 1: puntuaciones altas en todas las perspectivas (PP, PN, PH, PTF)

Cluster 2: puntuaciones bajas en $\mathrm{PH}$ y PN, altas en PP y puntuaciones medias en PTF

Cluster 3: puntuaciones medias en $\mathrm{PH}$ y $\mathrm{PN}$ y bajas puntuaciones en PP y PTF.

PANAS

(Medias)

Afecto positivo

Cluster $1 \overline{\mathrm{M}}=.74$

Cluster $2 \mathrm{M}=.78$

Cluster $3 \mathrm{M}=.69$
Afecto negativo

Cluster $1 \mathrm{M}=.48$

Cluster $2 \mathrm{M}=.41$

Cluster $3 \mathrm{M}=.49$

DSS WVS

Cluster $1 \mathrm{M}=.69$

Cluster $2 \mathrm{M}=.76$

Cluster $3 \mathrm{M}=.65$
Cluster $1 \mathrm{M}=.69$

Cluster $2 \mathrm{M}=.76$

Cluster $3 \mathrm{M}=.65$ 
Tabla 1

Análisis de resultados de la revisión sistematizada sobre la relación de la PT con factores socioeconómicos y psicológicos (31 textos). (Continuación)

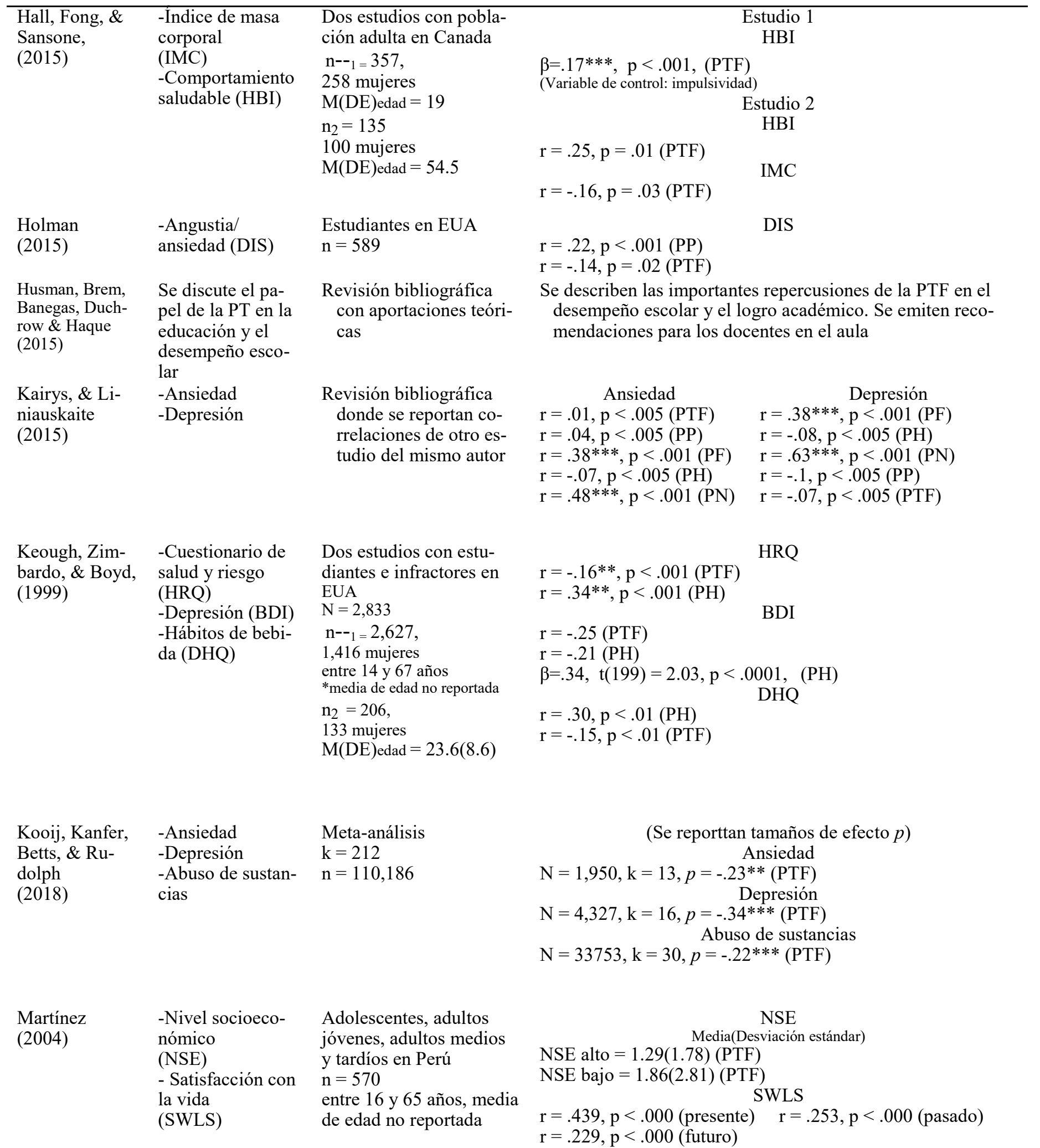


Tabla 1

Análisis de resultados de la revisión sistematizada sobre la relación de la PT con factores socioeconómicos y psicológicos (31 textos). (Continuación)

\begin{tabular}{|c|c|c|}
\hline $\begin{array}{l}\text { Padawer, Jacobs, } \\
\text { Hershey, \& } \\
\text { Thomas } \\
(2007)\end{array}$ & $\begin{array}{l}\text {-Variables demo- } \\
\text { gráficas } \\
\text { Edad } \\
\text { Sexo } \\
\text { Ingreso } \\
\text { Nivel educativo }\end{array}$ & $\begin{array}{l}\text { Adultos en EUA. Repre } \\
\text { sentatividad nac. } \\
\mathrm{n}=1,498 \\
784 \text { mujeres } \\
\mathrm{M}(\mathrm{DE}) \text { edad }=47.8(13.7\end{array}$ \\
\hline $\begin{array}{l}\text { Przepiorka \& So- } \\
\text { bol-Kwapinska } \\
(2020)\end{array}$ & $\begin{array}{l}\text {-Satisfacción c/ la } \\
\text { vida } \\
\text { (SWLS) }\end{array}$ & $\begin{array}{l}\text { Adultos en Polonia } \\
\text { reclutados con invita- } \\
\text { ción en línea } \\
\mathrm{n}=591 \\
51 \% \text { mujeres } \\
\text { M(DE)edad }=30(5.4)\end{array}$ \\
\hline
\end{tabular}

Seginer

(2017)

Stolarski \& Matthews (2016)

Stolarski, Zajenkowski, Jankowski \& Szymaniak (2020)

Zamora, Gil \& de Besa (2020)

Zimbardo \& Boyd (2015)

Zimbardo, Clements \& Rego (2017)
-Resultados acadé- Revisión bibliográfica micos

-Bienestar psicológico

-Satisfacción con la vida (SWLS)

-Numerosas variables psicológicas

-Cuestionario de persistencia universitaria (CPQ)

-Depresión (BDI) -Ansiedad (STAI) -Autoestima (EAR)

-Cuestionario de salud financiera con contribuciones teóricas

Estudiantes universitarios y sus familias en Polonia $\mathrm{n}=265$ adultos 143 mujeres

$\mathrm{M}(\mathrm{DE})$ edad $=22.5(3.3)$

Revisión sistemática con relación a variables psicológicas. Se analizan 41 estudios sobre PT balanceada

Estudiantes en España $\mathrm{n}=453$

263 mujeres

$\mathrm{M}(\mathrm{DE})$ edad $=19.7(4.7)$

Estudiantes en EUA $\mathrm{n}=205$

Medad $=23.6$

133 mujeres

Encuesta en línea en seis países: Brasil, EUA, China, Italia, Inglaterra y Alemania. $\mathrm{n}=3,000$

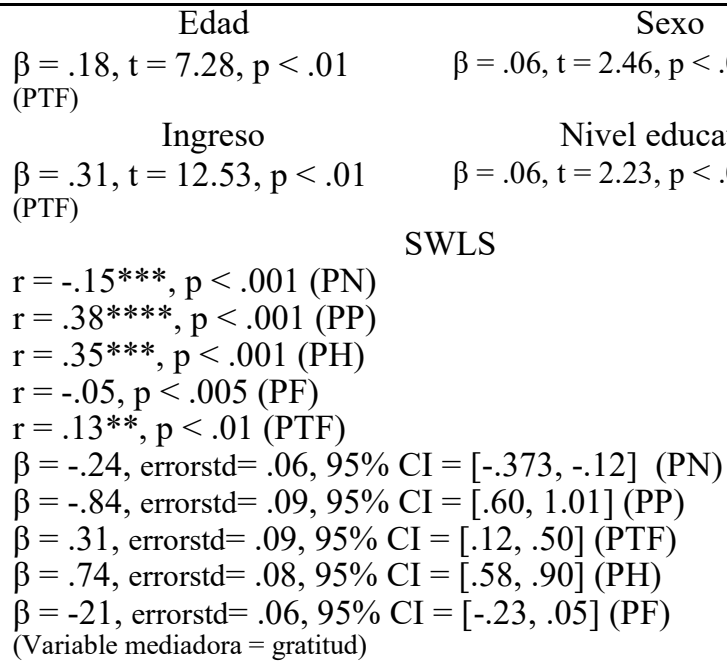

Se discuten los fundamentos teóricos de la relación direccional entre la PTF, el bienestar psicológico y el desempeño académico. Se concluye que a mayor PTF mejores resultados académicos.

$$
\begin{array}{ll}
\mathrm{r}=.20^{* *}, \mathrm{p}<.01(\mathrm{PP}) & \beta=.05, \mathrm{p}<.398(\mathrm{PF}) \\
\mathrm{r}=-.53^{* *}, \mathrm{p}<.01(\mathrm{PN}) & \beta=.01, \mathrm{p}<.872(\mathrm{PTF}) \\
\mathrm{r}=.11,(\mathrm{PH}) & \beta=.11, \mathrm{p}=.033(\mathrm{PP}) \\
\mathrm{r}=.07, \mathrm{p}(\mathrm{PTF}) & \beta=-.46, \mathrm{p}<.001(\mathrm{PP}) \\
\mathrm{r}=-.14^{*}, \mathrm{p}<.05(\mathrm{PF}) & \beta=.17, \mathrm{p}=.004(\mathrm{PH})
\end{array}
$$$$
\text { SWLS }
$$

Se analizan los resultados en torno a la medida DPTB, el cual resultó ser un predictor de numerosos comportamientos y actitudes determinantes de la calidad de vida.

\section{CPQ}

$\beta=.262, \mathrm{p}<.05$ (PTF)

$\mathrm{Mptf}=48.54(5.8), \mathrm{t}=5.78^{*}, \mathrm{~d}=.56-$ Grupo 1

$\mathrm{Mptf}=44.86(7.19), \mathrm{t}=5.78^{*}, \mathrm{~d}=.56-$ Grupo 2

Mnotas $=8.09(1.12), \mathrm{t}=7.51^{*}, \mathrm{~d}=.53-$ Grupo 1

Mnotas $=7.92(1.11), \mathrm{t}=7.51^{*}, \mathrm{~d}=.53-$ Grupo 2 BDI

STAI

$$
\begin{array}{cc}
\mathrm{r}=.59^{* * *}, \mathrm{p}<.001(\mathrm{PN}) & \mathrm{r}=-.25^{* * *}, \mathrm{p}<.001(\mathrm{PP}) \\
\mathrm{r}=.20^{* * *}, \mathrm{p}<00.1(\mathrm{PH}) & \mathrm{r}=.38^{* * *}, \mathrm{p}<001(\mathrm{PF}) \\
\mathrm{r}=. .19^{* *}, \mathrm{p}<.01(\mathrm{PTF}) & \mathrm{r}=.62^{* * *}, \mathrm{p}<.001(\mathrm{PN}) \\
\mathrm{r}=. .17^{*}, \mathrm{p}<.05(\mathrm{PP}) & \mathrm{r}=.07(\mathrm{PH}) \\
\mathrm{r}=.37^{* * *}, \mathrm{p}<.001(\mathrm{PF}) & \mathrm{r}=-.14^{*}, \mathrm{p}<.05(\mathrm{PTF}) \\
\multicolumn{1}{c}{\text { EAR }} &
\end{array}
$$$$
\mathrm{r}=-.48^{* * *}, \mathrm{p}<.001(\mathrm{PN})
$$$$
\mathrm{r}=.11(\mathrm{PH})
$$$$
\mathrm{r}=.13^{*}, \mathrm{p}<.05 \text { (PTF) }
$$$$
\mathrm{r}=.28^{* * *}, \mathrm{p}<.001(\mathrm{PP})
$$$$
\mathrm{r}=-.28^{* * *}, \mathrm{p}<.001(\mathrm{PF})
$$

Análisis cualitativo donde se concluye que:

-Personas con una visión negativa o positiva del pasado tienden a tener buena salud financiera.

-Personas con una visión fatalista o hedonista del presente tienden a tener una mala salud financiera.

-Personas con una alta orientación al futuro suelen tener una salud financiera inestable. 
Nota: STAI: Inventario de ansiedad estado-rasgo. EAR: Escala de autoestima de Rosenberg. CES-D: Escala de Depresión del Centro de Estudios Epidemiológicos. GME-Q: Cuestionario de experiencias sobre el monitoreo de glucosa. PANAS: Escala de afecto positivo y negativo. TSWLS: Escala de satisfacción temporal con la vida. SWLS: Escala de satisfacción con la vida. GDS: Escala de depresión geriátrica. SHS: Escala de felicidad subjetiva. WVS: Encuesta mundial de valores. DSS: Escala de síntomas depresivos (PNUD, 2012). HBI: Health behavior index. DIS: Entrevista de diagnóstico. HRQ: Cuestionario de salud y riesgo. BDI: Inventario de depresión de Beck. DHQ: Cuestionario de hábitos de bebida. CPQ: Cuestionario de persistencia en el colegio. Las siglas se basan en el nombre del instrumento en inglés.

Se incluyeron los valores $\beta$ en la casilla de correlaciones/efectos siempre que estos fueron reportados en cada estudio. En cada caso, los paréntesis de la derecha en la misma columna representan el marco temporal con que correlaciona la variable o su influencia en el caso de regresiones. Las abreviaciones PP, PN, PH, PF y PTF se explican en la introducción de este texto. En el caso de revisiones bibliográficas se presenta una síntesis cualitativa de las principales conclusiones. Algunas correlaciones se presentan por clusters o grupos según se hayan utilizado dichos análisis en cada estudio.

$* \mathrm{p}<0.05$

$* * \mathrm{p}<0.01$

$* * * \mathrm{p}<0.001$

De forma general, resaltan los libros: Time Perspective Theory; Review, Research and Application (Stolarski, et al., 2015) y Time Perspective. Theory and Practice (Kostić \& Chadee, 2017) publicados por Springer, los cuales giran en torno al enfoque desarrollado por el profesor Phillip Zimbardo, y presentan una serie de estudios orientados al desarrollo teórico y práctico de la PT.

De igual manera, se ubicaron algunos trabajos similares a éste. Por ejemplo, Stolarski, et al. (2020), realizaron una revisión sistemática de estudios que reportaron relación entre variables psicológicas y la medida de la desviación de la perspectiva temporal balanceada (DPTB; Stolarski, et al., 2011), la cual mide la diferencia entre una PT balanceada y la del individuo.

Aún cuando esta medida ha recibido críticas (McKay, et al., 2018), los autores resaltan su utilidad teórica y práctica. Mencionan que se trata de un constructo útil para predecir aspectos relacionados a la calidad de vida por encima de otras medidas, como la de personalidad. La revisión brinda una red nomológica extensa de esta medida con diversas variables demográficas, biológicas y psicológicas.

Una de las principales críticas en torno a esta medida, es la definición de los valores óptimos en cada perspectiva para establecer un balance, así como definir cual sería el su- puesto teórico que los soportaría. También, Jankowski, et al., (En revisión. Citado en Stolarski, et al., 2020) advierte que la DPTB asume asociaciones cuadráticas entre las diferentes perspectivas y las medidas de bienestar, mientras que otros estudios han evidenciado relaciones lineales.

Por su parte, Andre, et al., (2018), desarrollaron un metaanálisis de algunas medidas de orientación al futuro y sus relaciones con educación, trabajo y salud. Destaca que los efectos más generalizables fueron aquellos provenientes de medidas con dimensiones cognitivas, afectivas y del comportamiento. También la magnitud de las relaciones encontradas son comparables entre los dominios seleccionados.

A continuación, se discuten los resultados a partir de las variables de interés. El análisis gira en torno a la manera en que cada variable se relaciona con las diferentes perspectivas temporales. Los resultados obtenidos, dan muestra de la capacidad del constructo para brindar un panorama relativo a la calidad de vida de individuos o grupos sociales. Se finaliza discutiendo algunas implicaciones teóricas y prácticas en cuanto al concepto PT, así como se emiten algunas recomendaciones.

\section{Variables Socioeconómicas}

Para comprender como se relaciona la PT de 
una persona con su estatus socioeconómico, es necesario prever que esta relación se dá de forma indirecta. Es decir, cada marco temporal dominante representa un estilo de toma de decisiones y preferencias, que, a su vez, se traducen en un nivel de desempeño específico. Existen comportamientos de acuerdo a cada marco temporal que pueden favorecer o afectar los logros en educación, manejo del dinero y salud (Martínez, 2004; Padawer, et al., 2007).

\section{Educación}

Los procesos educativos y de aprendizaje representan la posibilidad de un mejor futuro. Desde el campo de la PT, se ha demostrado que personas que cuentan con una visión extensiva del futuro, pueden resistir mejor las tentaciones del entorno inmediato, y evitar así distracciones en la persecución de sus objetivos de largo plazo (Andre, et al., 2018; Husman \& Lens, 1999). Además, estudiantes con una extensiva PTF perciben más la instrumentalidad de sus acciones en el presente, lo que les permite alcanzar sus objetivos académicos (Husman, et al., 2015).

Desde la Teoría Social Cognitiva, la habilidad de planear el futuro y la percepción de autoeficacia para controlar los posibles eventos de la vida representan un aspecto fundamental de la agenciación humana (Bandura, 1997).

Bajo este enfoque, la PTF es indispensable para la motivación y el éxito académico. Por ejemplo, en un estudio de ZamoraMenéndez, et al. (2020), encontraron un efecto positivo de la PTF sobre la persistencia académica de estudiantes españoles $(\beta=.26$; $\mathrm{p}<.05$ ), sugiriendo, que aquellos orientados mayormente al futuro tienen más probabilidad de persistir en sus estudios, en comparación con sus pares orientados al presente.
No obstante de lo anterior, existen evidencias de que lo mismo sucede en sentido opuesto. Es decir, que el nivel educativo también puede influir en el desarrollo de una orientación hacia el futuro. Este es el argumento de Padawer, et al. (2007), quienes en un estudio de representatividad nacional en Estados Unidos, encontraron que el nivel educativo predijo la PTF de hombres jóvenes $(\beta=0.28, t[710]=$ $4.94, \mathrm{p}=0.01)$ y adultos $(\beta=0.11, t[710]=$ $2.18, \mathrm{p}=0.03)$. Una posible explicación de que el efecto sea mayor en hombres jóvenes, surge en torno a las diferencias en cómo planifican la vida de acuerdo a diferentes niveles educativos. Aquellos con un nivel alto de educación en la juventud, podrían aspirar a mejores posiciones ocupacionales, y es más probable que puedan encontrar oportunidades de desarrollarse socioeconómicamente. Este panorama de metas a largo plazo, puede que no esté tan presente en jóvenes con un menor nivel educativo, siendo su PTF más reducida.

En el mismo estudio, se reportó que el nivel educativo no predijo la PTF en el caso de las mujeres, lo que sugiere un mecanismo diferente para ellas. En el cual, el nivel educativo podría no tener tanto peso en comparación con otras variables como el ingreso o la edad (Padawer, et al., 2007).

Por su parte, Gonzalez-Lomelí, et al. (2017), compararon la PT de estudiantes universitarios en Colombia y México, diferencias significativas fueron encontradas entre ambos grupos de estudiantes en $\mathrm{PH}(t=3.965$, p $<.000)$ y $\mathrm{PN}(t=8.358, \mathrm{p}<.045)$, siendo los estudiantes colombianos los que puntuaron más alto en ambos factores. Esto implica que en comparación con los estudiantes mexicanos, ellos tienden a la impulsividad, son buscadores de riesgos, felices y despreocupados. $\mathrm{Y}$ por otro lado, tienden a centrarse demasiado en los eventos dolorosos de su pasado. 
Una recomendación de los autores, es seguir con el estudio de la PT en relación al desempeño académico. Con ello, se pueden atender mejor los procesos motivacionales de los estudiantes al ingresar a la universidad, e irlos orientando hacia sus metas a lo largo de su trayecto académico, fomentando este proceso a través de la orientación al futuro (Gonzalez-Lomelí, et al., 2017).

Al favorecer el desempeño escolar, la PTF puede también proveer de oportunidades laborales de mayor calidad, lo que se traduce consecuentemente en acceso a mejores viviendas, nutrición y oportunidades de desarrollo. También, la PTF encaja en un perfil de buen desempeño laboral, donde el empleado tiende a trabajar más y ser más eficiente, por lo tanto a ganar más (Kooij, et al. 2018; Gore, 2018).

Queda claro entonces, que una PTF puede resultar de un nivel educativo más alto, al mismo tiempo que puede influir en la obtención de este mismo. Aunque lo anterior parece sólo suceder en el caso de los hombres.

\section{Ingreso/finanzas personales.}

Al igual que con educación, hay ambigüedad acerca de la dirección que sigue la relación entre PT e ingreso. Algunos estudios plantean, que es la PT la que antecede el nivel de ingreso alcanzado y al estilo de manejo de recursos económicos; mientras otros, suponen que es la disponibilidad de este tipo de recursos la que promueve una mayor orientación al futuro (Fieulaine \& Apostolidis, 2015; Haushofer \& Fehr, 2014; Padawer, et al., 2007). No obstante, los resultados analizados sugieren una relación circular.

Resultados de un estudio de Zimbardo, et al. (2017), evidencian que la instrucción financiera no es suficiente para que una per- sona mantenga unas finanzas personales sanas, sino que es importante tener cierta orientación temporal. Con datos de 6 países y una muestra de más de 3,000 participantes, encontraron que la PT explicó mejor las decisiones financieras de los participantes, aún por encima de su capacidad matemática para calcular intereses compuestos. Con ello, se pone en duda el argumento que indica que la habilidad matemática antecede a unas buenas finanzas (Zimbardo, et al., 2017).

Otra conclusión que se desprende del estudio, es que personas con orientación al PN o PP, generalmente tienen buenas finanzas, y que esto puede deberse en parte, a que las personas orientadas al pasado de una forma negativa, tienden a ser extremamente precavidas o aversas al riesgo (Zimbardo \& Boyd, 1999). También, indican que aquellos orientados al presente son generalmente ineficientes en este aspecto. Particularmente los $\mathrm{PH}$ tienden a gastar, invertir y complacerse sin considerar consecuencias, mientras que los PF, tienden a sentirse atrapados e indiferentes ante el ahorro y el gasto (Klicperova, et al., 2015), por lo que ambos casos orientados al presente son los más propensos a tomar malas decisiones financieras.

Los autores no encontraron que las personas más orientadas al futuro tuvieran una mejor salud financiera. Sin embargo, los resultados sugieren que se perciben como individuos hábiles en el manejo de las finanzas sin que esto sea necesariamente cierto. Y puede deberse, según los autores, a una fuerte aspiración de éxito que puede conducir a toma de riesgos prometedores, pero que en la realidad no dan tantos beneficios. Mientras una persona orientada al pasado evitará tomar riesgos, otra orientada al futuro podría invertir en productos financieros que no comprende, o comprar cosas que no necesita. 
También Hall, et al. (2015), encontraron que la PT se relaciona al nivel socioeconómico (NSE), a través de una medida compuesta por las variables ingreso, categoría ocupacional y edad, sugiriendo mayor orientación al futuro en los estratos sociales más altos. Esto en una muestra de jóvenes universitarios en Estados Unidos. Aunque las correlaciones encontradas se ubican entre el tamaño pequeño y mediano de efecto (Cohen, 1988), apoyan el supuesto de relación entre PT y NSE. Cabe tener en cuenta que en este estudio se utilizó una medida que sólo considera el futuro, por lo que no queda claro el papel de otras orientaciones temporales en esta relación.

Desde otra perspectiva, la revisión que hacen Fieulaine y Apostolidis (2015), resalta cómo la pobreza y la inseguridad social moldean la PT de las personas, obligándolas a mantener su atención en cuestiones urgentes que requieren un gran esfuerzo cognitivo, entre las que destacan: resolver la precariedad de alimentos, la falta de vivienda o de servicios básicos, o protegerse de la intemperie.

Los autores mencionan la existencia de un nuevo panorama de la precariedad (a new landscape of precariousness; Nettleton \& Burrows, 2001, citado en Fieulaine \& Apostolidis, 2015), donde algunas personas no tienen la oportunidad de desarrollar los recursos cognitivos que les permitirían planificar más su vida, sobre todo cuando se vive bajo condiciones impredecibles o de vulnerabilidad, como en la pobreza. La precariedad de recursos económicos y sociales reduce la extensión del futuro subjetivo, y como consecuencia se adopta una de las perspectivas orientadas al presente (Adamkovič, \& Martončik, 2017; Lewin, 1942; Fieulaine \& Apostolidis, 2015).

En el mismo tenor, Eppel, et al. (1999), estudiaron la PT de personas sin hogar en
Estados Unidos, y descubrieron que aquellos que tenían menos tiempo en la indigencia mantenían una perspectiva más orientada al futuro, que los que tenían más tiempo (un mes). Esto, da indicios de que la intensidad o extensión de la pobreza también influye en la adopción de una PT, la cual difiere de la que se tiene cuando la disponibilidad de recursos no es problema.

También Gore (2018), realizó dos estudios donde investigó cómo influye la cantidad y estabilidad del ingreso en la PT. En el primero, 300 jóvenes adultos reportaron su ingreso personal y familiar, así como la estabilidad de éste durante el último mes, además de su PT. Los resultados evidenciaron que la estabilidad en el ingreso se vincula a una PT menos orientada al presente, sobre todo el PF. Esto significa que los participantes que percibieron tener un ingreso suficiente y estable resultaron los menos fatalistas (Gore, 2018).

El segundo estudio repitió la metodología del primero, pero en este participaron 167 adultos con un rango mayor de ingreso. Los resultados fueron similares, el ingreso y la estabilidad de éste resultaron vinculados a la PTF; y a la inversa, un ingreso más bajo e inestable predijo la orientación al presente.

Los resultados de Gore (2018), aportan evidencia de que la inestabilidad y la percepción de insuficiencia del ingreso, influye en el desarrollo de una perspectiva orientada hacia el presente. La estabilidad y disponibilidad de recursos, son factores clave para predecir como las personas responden a demandas del presente y del futuro. Cuando hay incertidumbre, lo más óptimo para el bienestar resulta centrar la atención en el presente y evitar una excesiva preocupación sobre el futuro (Eppel, et al., 1999; Fieulaine \& Apostolidis, 2015). Esto representa una ventaja 
momentánea que a la larga puede convertirse en un estilo habitual de manejo de recursos económicos (Zimbardo, et al, 2017).

\section{Salud.}

Muchos comportamientos que impactan la salud, se caracterizan por una disyuntiva entre el curso temporal de costos y beneficios. Hábitos como ejercitarse y llevar una alimentación sana, tienen beneficios a largo plazo, pero costos inmediatos.

Las investigaciones sobre el papel de la PT en la salud, se han centrado en los comportamientos que la favorecen o la afectan, relacionados con una orientación hacia el pasado, el presente o el futuro (Hall, et al., 2015; Stoltman, 2017; Thompson \& Fitzpatrick, 2007).

Consistente con la Teoría Social Cognitiva, individuos más orientados al futuro pueden anticipar mejor las consecuencias de sus actos, así como reconocer la instrumentalidad de sus acciones en el presente, para lograr beneficios en el futuro.

Al respecto, Hall, et al. (2015), encontraron que la orientación al futuro de jóvenes universitarios en EUA, predijo su tendencia a desarrollar comportamientos favorables para la salud, específicamente en: consumo de alcohol, uso del condón, higiene dental, dieta y uso del cinturón de seguridad $(\beta=.17, \mathrm{p}$ $<.001)$. En el mismo texto, los autores reportan un segundo estudio con el mismo enfoque pero con adultos tardíos. En este, la PTF correlacionó moderadamente con cuidado de la salud, a través de una medida compuesta por hábitos alimenticios $\mathrm{y}$ actividad física ( $\mathrm{r}$ $=.25, \mathrm{p}=.01)$.

Al mismo tiempo, los autores encontraron una correlación negativa entre Índice de Masa Corporal (IMC) y PTF $(r=-.16, p$
$=.03$ ), lo que sugiere que la orientación hacia el futuro también puede favorecer el control del peso, pues facilita la resistencia a tentaciones y permite apegarse a un plan establecido. Los análisis de regresión de los autores, mostraron un debilitamiento del $34 \%$ en la relación PTF-IMC cuando la variable cuidado de la salud fue controlada, convirtiendo la relación en no significativa $(\beta=-.11, \mathrm{t}=$ $-1.26, \mathrm{p}=.21$ ); con lo que se evidencia el papel mediador del cuidado de la salud en la relación PTF-IMC.

Con estos hallazgos, Hall, et al. (2015), confirmaron su hipótesis de que aquellos orientados hacia el futuro presentarían un mejor cuidado de la salud, en comparación con aquellos orientados al presente.

Los resultados tienen implicaciones teóricas y prácticas. Desde una perspectiva teórica, se evidencia el vínculo entre las diferentes orientaciones temporales con estados previos a la aparición de enfermedades. Y desde una perspectiva práctica, sería interesante comprender el riesgo de obesidad a través de sesgos en la PT. Es importante concebir la posibilidad de modificar esos sesgos

También, de acuerdo a sus resultados, los autores resaltan la importancia de mantener una perspectiva orientada al futuro durante la adultez temprana y tardía, con el fin de promover hábitos saludables (Adams \& Nettle, 2009; Andre, et al., 2018; Fieulaine, 2017; Kooij, et al., 2018).

Por su parte, Baird, et al. (2018), descubrieron que una perspectiva temporal balanceada (PTB; Boniwell \& Zimbardo, 2004), influye en la frecuencia con la que pacientes con diabetes tipo 1 monitorean su nivel de glucosa en la sangre. Lo que a su vez se traduce en un mejor control glucémico a largo plazo. Mientras otras investigaciones resaltan el beneficio de una PTF para el cuidado de la 
salud, los hallazgos de estos autores demuestran la conveniencia de ver la salud a través de un balance temporal. Esto es significativo, en el sentido de que, la influencia de la PT en el cuidado de la salud, es más complejo que sólo una orientación hacia el futuro. La perspectiva temporal balanceada (PTB), ha sido propuesta como un constructo que describe el perfil temporal más óptimo (Stolarski, et al., 2020).

Cabe tener en cuenta, que el balance de esta medida, basada en el modelo de Zimbardo y Boyd (1999), proviene de altas puntuaciones en PTF y PP, bajas en PF y PN, así como puntuaciones medias en $\mathrm{PH}$.

Una conclusión, que se desprende de Baird, et al. (2018), es que se podría trabajar en intervenir la PT de personas con diferentes enfermedades, con la intención de extender su perspectiva más hacia el futuro y menos hacia el presente. Esto con el objetivo de mejorar el apego a tratamientos, así como la propia percepción de la calidad de vida (Urzua et al., 2018). Por ejemplo, se ha desarrollado una terapia que se centra en modificar la PT de personas con Estrés Post Traumático (Zimbardo, et al., 2012). Esta terapia se centra en modificar la percepción negativa del pasado (PN) y el PF, e impulsar más el PP y la $\mathrm{PTF}$, así como el PH de manera moderada.

Con base en lo anterior, la PT puede plantearse como mediador en la relación entre salud y estatus socioeconómico, pues predice comportamientos que tienden a variar con el estatus socioeconómico y que impactan el estado de salud, (Guthrie, et al., 2009; Karelis, 2007).

En algunos estudios, las clases sociales más pobres muestran signos de orientación hacia el presente (Andre, et al., 2017; Eppel, et al., 1999; Fieulaine \& Apostolidis, 2015; Haushofer \& Fher, 2014; Karelis, 2007; Thompson \& Fitzpatrick, 2007), al mismo tiempo que presentan un estado de salud más deficiente (Hall, et al., 2015. Kooij, et al.
2018).

Para terminar el análisis de los factores socioeconómicos, cabe analizarlos en conjunto. Primeramente, es evidente que una orientación al futuro, y en particular una orientación temporal balanceada, pueden mejorar el estatus socioeconómico entendido a través del nivel y estabilidad del ingreso, el nivel educativo y el desempeño escolar, así como el cuidado de la salud. Una orientación al futuro como rasgo estable, permite al individuo vislumbrar y concientizar el futuro que quiere lograr, de manera que las decisiones en el presente favorecen el futuro.

Sin embargo, también está la cuestión de cómo las condiciones de vida en los diferentes estratos promueven la adopción de diferentes perspectivas temporales. A la luz de las evidencias presentadas hasta aquí, se percibe la desventaja que enfrentan los sectores más pobres de la sociedad, al verse sometidos a un estilo de vida que les demanda centrarse en el presente, teniendo como consecuencia malos desempeños en cuidado de la salud, en educación y en el manejo de recursos económicos. Este planteamiento encaja con lo que algunos autores han llamado trampa de la pobreza (poverty trap; Adamkovič, \& Martončik, 2017).

A continuación, el análisis de las variables psicológicas felicidad, satisfacción con la vida, afecto positivo-negativo, depresión $\mathrm{y}$ ansiedad en relación a la PT.

\section{Factores Psicológicos.}

En el caso de los factores psicológicos, el papel de la PTF suele ser menos claro que en el de los socioeconómicos. No obstante, existe mayor tendencia a encontrar correlaciones bajas o negativas entre este marco temporal y ciertos indicadores de bienestar, particularmente la felicidad. En el caso de las perspectivas del pasado y el presente, éstas tienen un papel importante en el bienestar subjetivo $\mathrm{y}$ 
psicológico, a través de una visión del mundo (o perspectiva) que puede aumentar o mermar el disfrute de la vida, así como dar origen a problemas más profundos, como la presencia de síntomas depresivos y la ansiedad.

\section{Felicidad y satisfacción con la vida.}

La literatura está dividida en cuanto a si es la orientación al pasado, presente o futuro la que tiene mayor peso en el bienestar subjetivo (Cunningham, et al., 2015; Przepiorka \& Sobol-Kwapinska, 2020).

En un estudio de Boniwell, et al. (2010), investigaron cómo los factores del ZTPI se relacionan con varias medidas de bienestar en Reino Unido y Rusia. Los resultados evidenciaron correlaciones moderadas con PN, PP y $\mathrm{PF}$, mientras que se presentaron correlaciones débiles con PH y PTF.

En la muestra británica, el PN tuvo sus mayores correlaciones con afecto negativo $(r$ $\left.=.46^{* * *}\right)$, así como las mayores correlaciones negativas con satisfacción con el pasado $(r=-.62)$. El PH presentó la mayor correlación positiva con una medida de bienestar eudaimónico $\left(r=.45^{* * *}\right)$; mientras que no se presentó ninguna correlación negativa significativa. El PTF solo tuvo una correlación significativa, esta fue negativa con una medida de actualización personal (Shostrom, 1963. Citado en Boniwell, et al., 2010). La mayor correlación positiva del PP, se dio con satisfacción con el pasado $(r=.52 * * *)$ y a la inversa con afecto positivo $(r=-.28 * * *)$. El PF sólo tuvo una correlación con afecto negativo $(r=.22 * *)$.

En la muestra de Rusia, se repitió el mismo procedimiento pero con medidas ligeramente distintas a las anteriores. En este caso, el PN tuvo sólo correlaciones negativas, siendo la mayor con felicidad $\left(r=-.51^{* * *}\right)$. El
PH no presentó correlaciones negativas, sólo una pequeña, con una medida de optimismo $\left(r=.20^{* *}\right)$. En el caso de PTF, tampoco hubo correlaciones negativas, y se presentó una correlación moderada con una medida de propósito de vida $\left(r=.36^{* * *}\right)$. El PP tampoco tuvo correlaciones negativas, pero si tuvo una correlación moderada positiva con felicidad $(r=.29 * * *)$. Finalmente, el PF sólo presentó correlaciones negativas, siendo la mayor con auto-eficacia $(r=-.30)$.

Una conclusión a la que llegan los autores, es que las correlaciones bajas entre PTF y algunas medidas de bienestar, se deben a la actuación de diferentes patrones temporales relacionados al balance en la PT (PTB). Personas con una fuerte orientación al futuro tienden a retrasar gratificaciones constantemente. Mientras que aquellos mas cercanos a la PT balanceada, la cual comprende el PH y el PP, además del PTF, pueden alcanzar mayor felicidad y satisfacción con la vida (Boniwell, et al., 2010).

Además, pareciera que la orientación hacia el futuro favorece sólo el bienestar eudaimónico. Otras versiones de bienestar más enfocadas al hedonismo no suelen verse favorecidas de una PTF (Boniwell, et al., 2010; Drake, et al., 2008).

La implicación en términos teóricos, es que las personas con una PTF dominante, llegan a sentir menos felicidad o satisfacción como resultado de frecuentes retrasos de gratificación, lo que podría disminuir el placer momentáneo en el día a día. Sin embargo, la posibilidad de poder influir en el futuro y poder alcanzar un plan deseado, puede favorecer la felicidad a largo plazo. En contraste, individuos orientados al PH o al PP, suelen sentirse más felices y satisfechos porque sus actividades habituales giran en torno al placer (Desmyter \& De Raedt, 2012; Boniwell, et 
al., 2010).

Se puede decir, que las estrategias cognitivas y de comportamiento se hacen evidentes, no solo en relación al futuro a través de una vida más activa, planeaciones y retraso de gratificaciones; sino también en relación al presente, a través de consentimientos placenteros o renuncias fatalistas. En el caso del pasado, las experiencias de vida forman una base de aprendizaje para elecciones futuras.

Por su parte, Cunningham, et al. (2015), realizan una revisión de literatura, en la que presentan relaciones consistentes entre PT y bienestar. Sintetizan su análisis proponiendo un modelo de dos vías para explicar la influencia de la PT en la felicidad y la satisfacción con la vida. La de arriba hacia abajo, es resultado de los procesos cognitivos de orden temporal que impactan el bienestar, relacionados a la manera como las personas interpretan el pasado y anticipan el futuro. Este efecto tiene lugar por medio de las emociones y opiniones relacionadas a las condiciones en que se vive, y no así a las condiciones mismas. Esto significa, que cuando las personas evalúan qué tan satisfechos con la vida se encuentran, lo hacen recolectando información de la memoria y tratando de anticipar sus experiencias.

De acuerdo con este planteamiento, se puede influir en el propio bienestar subjetivo acudiendo a recuerdos de manera consciente. Reviviendo momentos felices del pasado y tratando de reconciliarse o reinterpretar recuerdos negativos, dándoles un sentido de aprendizaje y experiencia (Cunningham, et al., 2015).

La otra vía, la de abajo hacia arriba, plantea que los rasgos de la personalidad del individuo influyen en sus condiciones de vida, pues a través de éstos se puede comprender la toma de decisiones y algunos comportamientos que anteceden al logro. Algunas decisio- nes tienen efectos inmediatos, mientras otras tienen efectos duraderos que pueden comprometer la felicidad en el futuro.

Finalmente, un aspecto positivo del $\mathrm{PH}$, es que correlaciona con bienestar subjetivo, afecto positivo y optimismo (Cunningham, et al., 2015), lo cual es beneficioso. No obstante, mucho disfrute y placer pueden fomentar la búsqueda de sensaciones y el abuso de sustancias, afectando la salud. Además las personas que son hedonistas tienden a ser más agresivos y depresivos (Zimbardo \& Boyd, 1999).

El PH representa un punto (teórico) intermedio en la PTB, es decir, no se considera completamente negativo ni positivo. Representa una perspectiva que debe intervenir en ocasiones para aliviar las reducciones de felicidad, producto de una perspectiva muy orientada al futuro, o de un periodo largo trabajando por cumplir las metas. Individuos felices pueden, a la larga, encontrar el balance temporal propuesto en la PTB. La acumulación de experiencias placenteras fomenta una visión más positiva de la vida, menos negativa del pasado, proyectando un futuro también positivo (Stolarski, et al., 2020).

\section{Afecto positivo/negativo (AP/AN) y autoesti- ma.}

Las principales correlaciones entre AP y el PH son casi de tamaño mediano según el criterio de Cohen (1988) ( $r$ 's entre .24 y .27). Lo mismo sucede con el PN ( $r$ 's entre -.21 y -.11), resultando correlaciones un poco más pequeñas y negativas comparadas con el $\mathrm{PH}$. En cuanto al AN, generalmente presenta correlaciones que tienden a ser altas con PN ( $r$ 's entre .38 y .56), y curiosamente suele haber pequeñas o negativas correlaciones con PTF ( $r$ 's entre .24 y -.21). Esto evidencia el difuso papel que juega el factor futuro en relación al bienestar subjetivo, particularmente con el 
balance afectivo. Aunque la PTF resulta fundamental para la motivación y la persecución de metas socioeconómicas, aún no queda muy claro el impacto que tiene en lo subjetivo (Cunningham, et al., 2015).

En cuanto a la autoestima, suele relacionarse con las categorías del pasado. Por ejemplo, Anagnostopoulos y Griva (2012), encontraron que el PP y el PN, se relacionan con la autoestima en una muestra de estudiantes en Grecia. El primero de forma positiva $(r=.19)$ y el último de forma negativa $(r$ $=-.57)$. Esto último sugiere que las experiencias de vida tienen un papel importante en la autoestima, produciéndose deterioros cuando la experiencia de lo vivido se percibe de forma aversiva. Sin embargo, varios estudios plantean que el PF es la perspectiva más relacionada a la baja autoestima (Boniwell, et al., 2010; Zimbardo \& Boyd, 2015).

Por otro lado, el PP puede ser un factor estrechamente relacionado a la "nostalgia", y puede ser que ésta misma promueva y mejore la autoestima, ayude a fortalecer los lazos relacionales y el apoyo social; además de brindar un significado a la vida, reforzando el valor de las tradiciones y rituales culturales, lo que a su vez aumenta el sentido de pertenencia cultural o incluso familiar.

Otros resultados sugieren que la edad es un factor mediador en la relación entre PT y bienestar subjetivo. Desmyter y De Raedt (2012), estudiaron la interacción de las medidas del ZTPI con la satisfacción con la vida, el balance afectivo y la depresión en una muestra de adultos mayores en Bélgica. En sus resultados, el afecto positivo pudo predecirse a través del $\mathrm{PH}(\beta=.34, t=3.61)$ y la PTF $(\beta=.32, t=3.17)$; mientras que el PP, demostró una pequeña influencia en la satisfacción con la vida $(\beta=.25, t=2.72)$.

Los autores analizaron sus hallazgos desde la Teoría de la Selectividad Socioemocional (TSS; Carstensen, et al., 1999), donde la percepción del tiempo juega un papel fundamental en el establecimiento de metas sociales, con implicaciones importantes para las emociones y la cognición a través de los cambios motivacionales que tienen lugar durante el desarrollo (Aquino, et al., 2017; Chen, et al., 2016; Desmyter y De Raedt, 2012).

De acuerdo con estos resultados, adultos mayores mejoran su bienestar subjetivo al enfocarse en el presente de una forma placentera (hedonista), al mismo tiempo que disfrutan de tener cierto control sobre su futuro.

En el mismo estudio, la perspectiva PN influyó en menos afecto positivo $(\beta=-.27, t$ $=5.28)$, más afecto negativo $(\beta=.31, t=$ $3.23)$, menos satisfacción con la vida $(\beta=-$ $.45, t=5.39)$, y más depresión $(\beta=.43, t=$ $5.28)$. También se encontró un efecto moderado de PF sobre depresión $(\beta=.29, t=$ 3.41).

Una visión positiva del pasado es fundamental para la satisfacción en la tercera edad, etapa en la cual la visión al futuro se ve más limitada. Según la TSS, cuando el tiempo se percibe como algo abierto, abundante, como en la juventud, se priorizan metas relacionadas al aprendizaje y la experiencia (futuro); pero cuando se percibe como algo limitado, como en la tercera edad, se priorizan metas emocionales (presente).

Una posible explicación para que el pasado tenga mayor peso que el futuro en la satisfacción con la vida, puede ser que no represente una referencia fácil de construir cognitivamente. Es decir, que es más sencillo acudir a la memoria, poner las emociones y pensamientos en algo ya vivido, que en algo que aún no sucede. El futuro debe ser anticipado; imaginado, no sucede lo mismo con el pasado. Sin embargo, puede ser que esto no suce- 
da cuando existe una condición de depresión o ansiedad (Anagnostopoulos \& Griva, 2012).

De acuerdo a estos resultados, personas de la tercera edad pueden aumentar sus emociones positivas, si se les brinda la oportunidad de que anticipen de alguna forma su futuro y hagan planificaciones de su vida, de una manera realista y alcanzable para ellos. Esto podría mejorar su balance afectivo y brindarles seguridad, al permitirles sentir más control sobre su vida.

\section{Depresión y ansiedad.}

Resultados del estudio realizado por Anagnostopoulos y Griva (2012), sugieren que una percepción negativa del pasado y una visión fatalista del presente, están relacionadas con la aparición de síntomas depresivos y con ansiedad. Los autores reportan que el PN presentó correlaciones considerables con síntomas depresivos $\left(r=.62^{* * *}\right)$ y ansiedad $(r$ $\left.=.77^{* *}\right)$. Mientras que el PF, presentó correlaciones moderadas con ambas medidas $(r$ $=.26^{* *}$ con depresión y $r=.33^{* *}$ con ansiedad). En el caso de la PTF no hubo correlaciones significativas.

Un aspecto a considerar, es que estas perspectivas pueden afectar otras orientaciones temporales. Cuando predomina una percepción negativa del pasado, pocos aspectos del presente pueden aportar placer de manera significativa. Lo que provoca que haya placer en menos ocasiones durante el día. También, cuando se adopta una actitud fatalista ante la vida, la tendencia a dejarse llevar y percibir la vida como algo que escapa a las capacidades personales, termina por reducir la visión sobre el futuro. En ambos casos, esto puede repercutir en la calidad y cantidad de relaciones interpersonales, resultando en interacciones mínimas e insatisfactorias (Zimbardo \& Boyd, 1999). Incluso, se ha comprobado que altas puntuaciones en PN y PF, pueden ser indicadores significativos de tendencia al suicidio en adolescentes (Laghi, et al. 2009).

Anteriormente, Zimbardo y Boyd (1999), ya habían evidenciado estas relaciones como resultado de pruebas de validez convergente y divergente del ZTPI. En su caso, también obtuvieron correlaciones altas de PN con depresión $(r=.59 * * *)$ y ansiedad $(r=.62 * * *)$. Al mismo tiempo, obtuvieron correlaciones moderadas de PF, también con depresión $(r$ $\left.=.37^{* * *}\right) \mathrm{y}$ ansiedad $\left(r=.38^{* * *}\right)$.

En algunos casos, el mecanismo de relación entre la PT y la salud mental, puede ser a la inversa de como se ha venido planteando. Es decir, no sólo la PT puede impactar en la salud mental, sino que la presencia de síntomas depresivos también puede influir en la manera cómo se percibe el pasado. Personas con síntomas depresivos tienden a ser selectivos en el tipo de humor que adoptan, prefiriendo la retracción y antipatía, esto ha llevado a ver la depresión como un trastorno relacionado al pasado (Drake, et al., 2008. Anagnostopoulos \& Griva 2012).

Estos resultados, en conjunto con los obtenidos para factores socioeconómicos, dan muestra de las implicaciones de la PT para la calidad de vida. Por un lado, cada orientación temporal promueve motivaciones, comportamientos, preferencias y toma de decisiones que en conjunto explican los niveles de desempeños en aspectos relacionados a la movilidad social, como educación, manejo de finanzas y cuidado de la salud.

Además, se percibe una relación circular de la PT con los factores aquí analizados, en el sentido de que, de acuerdo a cada perspectiva, se puede alcanzar cierto nivel socioeconómico, a la vez que cada nivel socioeconómico promueve perspectivas específicas.

Y por otro lado, cada PT tiene sus repercusiones en la salud mental y el bienestar 
subjetivo. Donde las perspectivas del pasado parecen estar fuertemente relacionadas a la felicidad y la satisfacción con la vida. Como se vio en esta revisión.

La manera positiva o negativa con la que se recuerda el pasado, ayuda a comprender los niveles de bienestar subjetivo de una persona en el presente. Al mismo tiempo, personas con diferentes niveles de bienestar desarrollan diferentes perspectivas temporales. Siendo las perspectivas negativas del pasado y el presente las que mayores efectos presentan.

En cuanto al futuro, parece ser que, cuando se concibe con un sentido de diligencia planificación o agencia, como en el modelo de Zimbardo y Boyd (1999), sólo mejora el bienestar a través de una PTB. Es decir, cuando el perfil temporal completo abarca también el PP y el PH.

Al respecto, Urzúa, et al. (2018), descubrieron que la idea que se tiene del futuro juega un papel importante en las estimaciones subjetivas de calidad de vida, ya que las personas acuñan un patrón de comparación temporal entre un estado actual y uno ideal. Es decir, que las personas se comparan con lo que les gustaría ser o tener en el futuro, o con lo que han tenido antes comparado con lo que tienen ahora.

\section{Discusión}

Los resultados obtenidos permiten apreciar que se trata de un fenómeno fundamental para el funcionamiento humano, que tiene grandes implicaciones en la calidad de vida de las personas. El gran número de trabajos sobre el tema, da muestra del interés que ha despertado en investigadores del área de la Psicología y las Ciencias Sociales.

Queda claro que cada perspectiva sirve de marco de referencia para el ejercicio, no siempre consciente, de un estilo cognitivoconductual que promueve pensamientos, emociones, actitudes y comportamientos que ayudan a darle un sentido al mundo, por medio del cual se justifican decisiones, niveles de desempeño y de logro. Las evidencias establecen que la PT ejerce un rol fundamental en lo que una persona puede llegar a tener y ser.

Al considerar la calidad de vida como un fenómeno compuesto tanto de elementos objetivos (socioeconómicos) como subjetivos (psicológicos) (Campbell, et al., 1976; Cummins, 2005; Urzúa \& Caqueo-Urizar, 2012; Urzúa, et al., 2018; Veenhoven, 2006), se puede pensar en la orientación al futuro como un aspecto que puede favorecer en lo objetivo, pero hasta cierto punto afectar en lo subjetivo. Es decir, mantener una perspectiva orientada hacia el futuro puede servir para un manejo más diligente de los recursos, un mejor desempeño académico y mejor cuidado de la salud; pero al mismo tiempo puede promover una excesiva atención en metas y planes de largo plazo, afectando la capacidad de "vivir el presente", reduciendo el disfrute y la felicidad.

Por otro lado, el hecho de que los sectores más necesitados de la sociedad vivan en un entorno que termina por "estancar" su atención en el presente, sugiere que ellos deben enfrentar vulnerabilidades de las que no son conscientes, y que repercuten en la forma como se desempeñan en áreas importantes como el cuidado de la salud, la educación y el manejo de recursos.

Claro que la pobreza no se acaba con orientarse al futuro, pero extender la PT de quienes sufren la pobreza puede representar una parte complementaria para la política pública enfocada en el bienestar. Esto podría ayudar a que desarrollen habilidades para crear alternativas que les permitan perseguir el estilo de vida que tengan motivos para valorar. Un estilo de vida más orientado a quien se quiere ser en el futuro, o más enfocado al 
logro de metas. Claro que para que esto sea posible, cambios socioestructurales deben suceder primero.

De acuerdo con la orientación al presente, se promueven estilos cognitivo-conductuales que indirectamente afectan la $\mathrm{CV}$ en su parte objetiva (o socioeconómica), pero que a su vez promueven altos índices de bienestar subjetivo, siguiendo una lógica del tipo "pobres pero felices" (Ochoa, 2011; Rojas, 2005).

En resumen, se puede concluir, que la PT puede ser útil para estimar la $\mathrm{CV}$ de personas y sociedades, ya que, con base en los resultados aquí presentados, se puede estimar la situación objetiva-subjetiva con base en una PT dominante. Para ello, se debe tener en cuenta como actúa cada marco temporal, sobre todo en el caso de las orientaciones PTF y PF-PH, que forman dos extremos de un continuo que pudiera representar la $\mathrm{CV}$, en su punto más alto y más bajo respectivamente. Se debe tener en cuenta, que también el $\mathrm{PH}$ debe tomar un rol importante en ese continuo, por sus contribuciones a la felicidad y el disfrute del presente.

No obstante, el enfoque aquí propuesto podría ayudar a comprender por qué en un país como México (o en cualquier otro de Latinoamérica), donde casi la mitad de la población vive en pobreza extrema, se reporta cada vez mayor satisfacción con la vida (OCDE, 2020). Esto puede deberse a que la perspectiva dominante en el país sea el $\mathrm{PH}$. No obstante, es necesaria una investigación de alcance nacional para descubrir el perfil temporal de la sociedad mexicana, y poder contrastarla con su CV.

Integrar PT y CV, representa un intento conceptual-metodológico por abordar cuestiones que hasta ahora no han podido ser explicadas por la economía tradicional (Thornton, 2017). Una propuesta proviene del campo de la economía conductual, la cual conjunta el que hacer de la economía y la psicología (Camerer \& Lowenstein, 2004). Al respecto, Muñoz-Munguía, et al., (2019), realizan una síntesis del surgimiento, evolución y las aplicaciones a diversos campos de las ciencias sociales, con énfasis en el desarrollo regional. En ese sentido, ha sido posible incorporar las dimensiones del comportamiento humano (Ej. cognición, emoción e instrumental) al estudio de la pobreza económica o patrimonial, y con ello, explicar los cambios que ocurren a nivel subjetivo.

Para finalizar, se podría aprovechar la llamada Terapia de Perspectiva Temporal utilizada en personas con Estrés Post Traumático (Zimbardo, et al., 2012), con el fin de transformar la PT de grupos vulnerables, hacia otra más positiva y tal vez extensiva. Esto permitiría enfocar su visión en un límite razonable de acuerdo a sus posibilidades, y a las que el sistema estructural permita y promueva.

En ese sentido, la propuesta de transformar la PT no pretende ser una posible solución objetiva al problema de la pobreza, sino una parte complementaria, que, entre otras cosas, podría ayudar a la política pública a medir el impacto de sus estrategias a un nivel psicológico, incluso cuando su asistencia no gire en torno a ello.

Intervenir la PT de beneficiarios de programas, al mismo tiempo que reciben asistencia, permitiría extender más hacia el futuro la PT que si no se hiciera en conjunto. Por ejemplo, sí programas de despensa pueden aliviar la preocupación de una familia por unos días, cada mes, la transformación hacia una PTF permitiría que por lo menos en ese lapso, la persona planifique metas alcanzables, y adquiera una visión más positiva por lo menos de esos días. Se trataría de mejorar los recursos subjetivos, al mismo tiempo que se mejoran los objetivos.

Claro que para lograrlo, es fundamental y 
urgente cambiar las condiciones estructurales que excluyen a los pobres, les restan oportunidades y los discriminan (Guzmán-Correa, 2017; Palomar \& Lanzagorta, 2005). Para que tenga sentido orientarse al futuro, no solo deben abrirse oportunidades específicas para ellos en sentido objetivo, tales cómo trabajo, acceso a servicios y asistencias directas, sino que también debe trabajarse por brindar seguridad subjetiva a los más necesitados, con el fin de hacer válido su derecho a la tranquilidad, que les permita soñar con un futuro alcanzable.

\section{Conclusiones}

La presente revisión reúne un número considerable de correlatos que permiten ubicar patrones teóricos que enriquecen el estudio de la PT. Cabe mencionar que el análisis de estos patrones fue guiado a través de las variables consideradas, más que en lugar de los participantes y sus características.

El objetivo del artículo fue evidenciar como la PT se relaciona con factores socioeconómicos y psicológicos con el fin de evidenciar su vínculo con la calidad de vida, en su parte objetiva y subjetiva (Cummins, 2005; Urzúa, et al., 2018).

La PT impacta indirectamente la parte objetiva (o socioeconómica) de la calidad de vida, de forma parecida a lo señalado por Cunningham en su modelo de la vía de abajo hacia arriba (Cunningham, et al., 2015). En la cual, la PT entendida como un aspecto de la personalidad, produce niveles de desempeño que posteriormente se traducen en la posición social del individuo.

En este sentido, el ZTPI ha resultado útil en definir un perfil temporal orientado al futuro en términos de diligencia, planificación y capacidad de agencia. Los resultados obtenidos a través de esta medida, en relación al futuro, suponen que quienes viven mayormente bajo esta perspectiva son más organi- zados, responsables y hábiles para perseguir sus objetivos.

Sin embargo, la medida sólo concibe la parte positiva del futuro, donde no cabe la posibilidad de que este se perciba como algo negativo, que a su vez pueda influir en dificultades para el desarrollo de capacidades de agencia. Cabe señalar, que algunas propuestas han demostrado lo innecesario que resulta proponer una parte negativa del futuro en el modelo de Zimbardo y Boyd (1999) (KoŠt'ál, et al., 2015), faltan más estudios en diferentes países y con otros participantes, diferentes a estudiantes universitarios.

Teniendo esto en consideración, la subescala PTF del ZTPI, puede ser útil para ubicar fortalezas de movilidad social. En el sentido de que altas puntuaciones están relacionadas a una mayor capacidad de agencia.

Es importante señalar diferencias metodológicas entre estudios que utilizan un mismo enfoque. Principalmente se ha criticado la proliferación de versiones cortas del ZTPI, suscitando algunas propuestas para la revisión de los ítems más robustos del instrumento (Peng, et al., 2021). Ya que en algunos casos las estructuras varían mucho de acuerdo a la selección de ítems para formar modelos, aun cuando en teoría se están midiendo los mismos factores en todas las investigaciones que utilizan el instrumento.

También se necesita profundizar en cómo es que la edad y el género se relacionan con la PT de forma empírica. Aunque teóricamente hay una base sólida que puede servir para explicar esto, los resultados en las investigaciones no son tan consistentes como se propone. Ya que, mientras algunos estudios no encuentran diferencias significativas a través de estas variables, otros llegan a plantearlas como posible causa de la PT (Chen, et al, 2016; Mello, 2019).

La habilidad para planear el futuro, relacionada a la percepción de autoeficacia para 
controlar los posibles eventos de la vida, representan un aspecto fundamental de la agenciación. Sin embargo, va más allá de pensar sobre el futuro y planear metas. Al tratarse de un fenómeno que no siempre es consciente, puede ser que escape a la capacidad personal. El desarrollo de una orientación al futuro no solo requiere de esfuerzo, sino también, que las condiciones y la estructura de la vida diaria brinden sentido a esta posibilidad.

Cuando las condiciones son precarias, asumir una orientación hacia el presente resulta lo más adaptativo (Fiaulaine \& Apostolidis, 2015). A su vez, la adopción de estas perspectivas afecta el desempeño en educación, cuidado de la salud y manejo de finanzas. Lo que representa un riesgo para la calidad de vida. Esto sugiere una relación circular que podría representar lo que algunos investigadores llaman "trampa de la pobreza" (Adamkovič, \& Martončik, 2017).

Aún cuando se ha manejado como un problema económico, conocer la psicología de la pobreza y de quien la sufre, es también de vital importancia, tanto en sus repercusiones, como en los posibles factores causales de orden subjetivo (Fell \& Hewstone, 2015).

Para Sen (2000), la pobreza es la privación de capacidades básicas que permiten a las personas tener la oportunidad de evitar el hambre, la desnutrición, la carencia de vivienda, o de ser capaces de tomar parte en la sociedad y la vida de la comunidad. Numerosas evidencias respaldan el supuesto de que la orientación al futuro podría representar una capacidad ese tipo.

También es importante comprender que el hecho de tener que dirigir toda la atención hacia el presente, aumenta la vulnerabilidad de quienes sufren la pobreza. Ya que influye en un manejo menos eficiente del cuidado de la salud y los recursos. La vulnerabilidad se refiere al aumento en la fragilidad, relacionada a la indefensión y a la carencia de recursos para enfrentar adversidades. Las cuales en ocasiones deben ser anticipadas. Para lo cual la orientación hacia el futuro es necesaria.

Teniendo en cuenta lo anterior, es importante ubicar qué sectores de la sociedad actúan habitualmente bajo una perspectiva poco orientada al futuro, ya que son más vulnerables a tomar malas decisiones financieras, así como hacer mal uso de sus recursos socioeconómicos, aumentando así la incertidumbre y la perdida de claridad sobre el futuro.

Por otro lado, la parte subjetiva de la calidad de vida se relaciona principalmente con las orientaciones al pasado, y en segundo lugar al presente. Estas perspectivas influyen directamente en la percepción subjetiva, favoreciendo un estado interno del ser por medio del cual puede aumentar o disminuir el disfrute de la vida. Aumentando cuando se adopta una perspectiva PP o PH, y disminuyendo con PN y PF. El hecho de que la PTF presente correlaciones débiles (o nulas) con felicidad, sugiere que lo más óptimo es un equilibrio entre esfuerzo y disfrute, en lugar de ambicionar el logro incesante de metas.

Falta definir si la PT es algo estable o disposicional. Es decir, si es un rasgo fijo en la personalidad del individuo, o si este acude a diferentes perspectivas según la situación y de acuerdo a su propio criterio. O bien, una tercera opción, es que sea la situación o el contexto mismo el que influye en una PT generando diferentes niveles de respuestas en distintos individuos.

Cuando se considera un aspecto disposicional, la explicación de diferencias individuales en este sentido, podría girar en torno a que haya niveles en la habilidad para cambiar de PT, significando que unas personas pueden ir más fácil del $\mathrm{PH}$ al PP y al PTF, que otras.

Finalmente, aunque se plantea que la PT depende del estatus socioeconómico, también se ha dicho que podría ser causa del mis- 
mo, concluir en cualquiera de estas dos aseveraciones parece inadecuado todavía. La mayoría de los estudios son a nivel de correlación, por lo que hacen falta mas estudios de corte explicativo para ubicar si la PT causa el nivel socioeconómico, o al revés. No obstante, es muy probable que se trate de una relación circular.

Lo ideal sería que la PT tenga un sentido funcional y que además sea posible de entrenar, sobre todo en los contextos donde generalmente se mantienen orientados al presente, en cualquiera de sus versiones (fatalista o hedonista). Debe ser posible crear las condiciones para que las personas tengan mayores oportunidades de construir su futuro, sean más conscientes de él y obtengan las herramientas y condiciones para lograrlo. De forma que se pueda ayudar a las personas a perseguir el tipo de vida que desean.

\section{Limitaciones}

Una limitante de la revisión fue la selección arbitraria de variables, en el sentido de que pudieran tomarse otros indicadores diferentes a los aquí considerados para evaluar cuidado de la salud, nivel socioeconómico y educación. En este caso, la selección de textos, posterior al análisis de los más de 1,000 resultados iniciales, correspondió más al criterio del primer autor del artículo.

También, otras revisiones pudieran enfocarse a metodologías específicas. Por ejemplo, limitándose a estudios que hayan utilizado el mismo instrumento, o incluso reunir solo resultados de estudios longitudinales o con grupos de control. Esto último pudiera resultar provechoso considerando que predominan estudios correlacionales, cuyas aportaciones alcanzan a llegar sólo hasta el nivel teórico. Tal es el caso de la presente revisión.

Otro aspecto a considerar, es que algunos estudios consideran solamente el futuro como dimensión temporal única de análisis, dejando fuera el pasado y el presente. Es importante profundizar más allá de la consideración aislada del futuro.

También, la mayoría de estudios giran en torno a la medida del ZTPI. Aún cuando se presentan algunos estudios que consideran el tiempo subjetivo en términos unidimensionales (únicamente al futuro), la mayor parte del análisis gira en torno al modelo teórico del mismo instrumento. Esto debido a la abundancia de investigaciones que utilizan este modelo y a la consistencia que guardan los resultados obtenidos. Otras revisiones pudieran dar mayor peso a otros enfoques.

Finalmente, en gran parte de los casos se acudió a muestras de estudiantes universitarios. No obstante que hay estudios que si utilizan otro tipo de participantes, estos son muchos menos que los desarrollados con estudiantes.

\section{Agradecimientos}

Reconocimiento de subsidios: la elaboración del presente artículo fue posible gracias al Consejo Nacional de Ciencia y Tecnología de México (CONACYT), quien otorgó una beca para estudios doctorales al primer autor.

\section{Referencias}

Adams, J., \& Nettle, D. (2009). Time perspective, personality and smoking, body mass, and physical activity: An empirical study. British Journal of Health Psychology, 14, 83-105. https:// doi.org/10.1348/135910708X299664

Adamkovič, M., \& Martončik, M. (2017). A review of consequences of poverty on economic decisionmaking: A hypothesized model of a cognitive mechanism. Frontiers in Psychology, 8(OCT), 1 -13. https://doi.org/10.3389/fpsyg.2017.01784.

Anagnostopoulos F., \& Griva, F. (2012). Exploring Time Perspective in Greek Young Adults: Validation of the Zimbardo Time Perspective Inventory and Relationships with Mental Health Indicators. Social Indicators Research, 106, 41-59. 
https://doi.org/10.1007/s11205-011-9792-y

Andre, L., van Vianen, A. E. M., Peetsma, T. T. D., \& Oort, F. J. (2018). Motivational power of future time perspective: Meta-analyses in education, work, and healh. PloS ONE, 13(1), 1-45. https://doi.org/10.1371/ journal.pone.0190492

Aquino, T. A. A., Gouveia, V. V., Gomes, E. S., \& Melo de Sá, L. B. (2017). La percepción de sentido de la vida en el ciclo vital: una perspectiva temporal. Avances en Psicología Latinoamericana, 35(2), 375-386. doi: http:// dx.doi.org/10.12804/revistas.urosario.edu.co/ apl/a.3728

Baird, H. M., Webb, T. L., Martin, J., \& Sirois, F. M. (2018). The Relationship Between a Balanced Time Perspective and Self-monitoring of Blood Glucose Among People With Type 1 Diabetes. Annals of behavioral medicine: a publication of the Society of Behavioral Medicine, 53(2), 196 -209. https://doi.org/10.1093/abm/kay028

Bandura, A. (1997). Self-efficacy: The Exercise of Control. New York: W.H. Freeman \& Company

Boniwell, I., \& Zimbardo, P. (2004). Balancing time perspective in pursuit of optimal functioning. En P. A. Linley \& S. Joseph (Eds.), Positive psychology in practice, (pp. 165-179). Wiley. https://doi.org/10.1002/9781118996874.ch13

Camerer, F. C., \& Lowenstein, G. (2004). Behavioral Economics: Past, Present, Future. En C. F. Camerer, G. Lowenstein, \& M. Rabin (Eds.), Advances in Behavioral Economics, (pp. 1-51). Princeton University Press.

Campbell, A., Converse, P. E., \& Rogers, W. L. (1976). The Quality of American Life: Perceptions, Evaluations and Satisfaction. RussellSage.

Carstensen, L. L., Isaacowitz, D. M., \& Charles, S. T. (1999). Taking Time Seriously: A Theory of Socioemotional Selectivity. A merican Psychologist, March;54(3):165-81. doi: 10.1037//0003-066x.54.3.165

Chen T., Liu L, Cui J., Chen X., \& Wang Y. (2016). Developmental Trajectory of Time Perspective: from Children to Older Adults. Psych Journal, 5(4):245-255. https://doi.org/10.1002/pchj.140

Chen, T., Lui, L., Cui, J., Chen, X., Wang, J., Zhang, Y., Wang, Y., Li, X., Neumann, D., Shum, D. H. K., Wang, Y., \& Chan, R. C. K. (2016). Present-Fatalistic Time Perspective and Life Satisfaction: The Moderating Role of Age. Personality and Individual Differences, 99, 161165.
Codina, L. (2018). Revisiones bibliográficas sistematizadas: procedimientos generales y Framework para ciencias humanas y sociales. Barcelona: Universidat Pompeu Fabra. http:// hdl.handle.net/10230/34497

Cohen, J. (1988). Statistical Power Analysis for the Behavioral Sciences (2da ed.). Lawrence Erlbaum Associates, Publishers. http:// www.utstat.toronto.edu/ brunner/ oldclass/378f16/readings/CohenPower.pdf

Cummins, R. A. (2005). Moving From the Quality of Life Concept to a Theory. Journal of Intellectual Disability Research, Octubre(49), 699-706. https://doi.org/10.1111/j.13652788.2005.00738.x

Cunningham, K. F., Zhang, J. W., \& Howell, R., T. (2015). Time perspectives and Subjective WellBeing: A Dual-Pathway Frame-Work. En Stolarski, Fieulaine \& Van Beek (Eds.), Time Perspective Theory; Review, Research and Application. Essays in honor of Phillip Zimbardo. (pp. 403-418). Springer. DOI: 10.1007/978-3319-07368-2_26

Desmyter, F., \& De Raedt, R. (2012). The Relationship Between Time Perspective and Subjective Well-being of Older Adults. Psychologica Belgica, 52(1), 19-38. DOI: 10.5334/pb-52-1-19

Drake, L., Duncan, E., Sutherland, F., Abernethy, C., \& Henry, C. (2008). Time Perspective and Correlates of Wellbeing. Time and Society, 17(1), 47-61. DOI: 10.1177/0961463X07086304

Eppel, S. E., Bandura, A., \& Zimbardo, P. G. (1999). Escaping homelessness: The Influences of Selfefficacy and Time Perspective on Coping with Homelessness. Journal of Applied Social Psychology, 29, 575-596. https://doi.org/10.1111/ j.1559-1816.1999.tb01402

Fell, B., \& Hewstone, M. (2015). Psychological Perspectives on Poverty. Joseph Rowntree Foundation. https://www.jrf.org.uk/report/ psychological-perspectives-poverty

Fieulaine, N., (2017). Time Perspective and Cannabis Use: Why and How It Is More Complex Than What We Think. En A. Kostić \& D. Chadee (Eds.), Time Perspective: Theory and Practice, (pp. 195-217). Palgrave Macmillan. https:// doi.org/10.1057/978-1-137-60191-9

Fieulaine, N., \& Apostolidis, T., (2015). Precariousness As a Time Horizon: How Poverty and Social Insecurity Shape Individuals' Time Perspectives. En M. Stolarski, N. Fieulaine, \& W. van Beek (Eds.), Time Perspective Theory; Review, Research and Application. Essays in honor of Phillip Zimbardo, (pp. 213-230). 
Springer. $\quad$ https://doi.org/10.1007/978-3-31907368-2

Frankl, V. E. (1988). La voluntad de sentido. Barcelona: Herder.

González Lomelí, D., Maytorena, M. Á., Lohr Escalante, F. \& Carreño Cruz, E. A. (2006). Influencia de la perspectiva temporal y la morosidad académica en estudiantes universitarios. Revista Colombiana de Psicología, (15),15-24. https:// www.redalyc.org/pdf/804/80401503.pdf

Gonzalez Lomelí, D., Maytorena, M. Á., Cárdenas, N. \& Tapia, C. (2017). Perspectiva temporal de estudiantes universitarios mexicanos y colombianos. Revista Iberoamericana de Diagnóstico $y$ Evaluación - e Avaliação Psicológica, RIDEP, 1(No.46), 133-145. https://aidep.org/ sites/default/files/2018-01/RIDEP46-Art10.pdf

Gore, (2018). Standing on shaky ground and living in the now: How resource amount and stability influence time perspectives. Journal of Applied Psychology, 2018(48), 398-408. https:// doi.org/10.1111/jasp.12520

Güel, P., Orchard, M., Yopo, M. \& Jiménez-Molina, A. (2015). Time perspective and subjetctive well-being in Chile. Social Indicators Research, 123, 127-141. https://doi.org/10.1007/s11205014-0727-2

Guthrie, L. C., Butler, S. C., \& Ward, M. M. (2009). Time perspective and socioeconómic status: a link to socioeconómic disparities in health? Social Science and Medicine, (68), 2146-2151. https://doi.org/10.1016/ j.socscimed.2009.04.004

Guzmán Correa, P. R (2017). La pobreza y desigualdad en la Ciudad de México, el caso de la pobreza alimentaria. Instituto de Investigaciones Parlamentarias. Asamblea legislativa del Distrito Federal, VII Legislatura. http:// www.aldf.gob.mx/archivo386e2d7d4b4497878bc446e7f3d46fbc.pdf

Hall, P. A., Fong, G. T., \& Sansone, G. (2015). Time Perspective As a Predictor of Healthy Behaviors and Disease-Mediating States. En M. Stolarski, N. Fieulaine \& W. van Beek (Eds.), Time Perspective Theory; Review, Research and Application. Essays in honor of Phillip Zimbardo, (pp 117). Springer.

Haushofer, J., \& Fehr, E. (2014). On the psychology of poverty. Science, 344, 862-867. https:// doi.org/10.1126/science.1232491

Husman, J., Brem, S. K., Banegas, S., Duchrow D. W., \& Haque, S. (2015). Learning and future time perspective: the Promise of the future - rewarding in the present. En M. Stolarski, N. Fieulaine \& W. van Beek (Eds.), Time Perspective Theory; Review, Research and Application. Essays in honor of Phillip Zimbardo. (Pp 131143). Springer. https://doi.org/10.1007/978-3319-07368-2_8.

Husman, J., \& Lens, W. (1999). The role of the future in student motivation. Educational Psychologist, 34(2), 113-125. https://doi.org/10.1207/ s15326985ep3402_4

International Time Perspective Network (2020, 17 abril). Before and After: Developmental Aspects of Time [Video]. Youtube. https:// www.youtube.com/watch? $=8$ XCGtila4b0

James, W. (1989). Principios de Psicología. Fondo de Cultura Económica.

Kairys, A., \& Liniauskaite, A. (2015). Time perspective and personality. En M. Stolarski, N. Fieulaine \& W. van Beek (Eds.), Time Perspective Theory; Review, Research and Application. Essays in honor of Phillip Zimbardo. (pp 99115). Springer. https://doi.org/10.1007/978-3319-07368-2_6

Karelis, C. (2007). The persistance of poverty. Why the economics of the well-off can't help the poor. Yale University Press.

Keough, K. A., Zimbardo, P. G., \& Boyd, J. N. (1999). Who's smoking, drinking, and using drugs? Time perspective as a predictor of substance use. Basic and Applied Social Psychology, 21, 149-164. https://doi.org/10.1207/ S15324834BA210207

Klicperova, M., Košt’ál, J., \& Vinopa J. (2015). Time perspective in consumer behavior. En: M. Stolarski, N. Fieulaine, \& W. van Beek (Eds.), Time Perspective Theory; Review, Research and Application, (pp. 17-56). Springer. DOI 10.1007/978-3-319-07368-2_23,

Kooij, D. T. A. M., Kanfer, R., Betts, M., \& Rudolph, C. W. (2018). Future Time Perspective: A Systematic Review and Meta-Analysis. Journal of Applied Psychology, 103(8), 867-893. https:// doi.org/10.1037/ap10000306

KoŠt'ál, J., Klicperová-Baker, M., \& Lukavsky, J. (2015). Short version of the Zimbardo Time Perspective Inventory (ZTPI-short) with and without Future-Negative Scale, verified on nationally representative samples. Time and Society, 0(0), 1-24. https://journals.sagepub.com/ doi/10.1177/0961463X15577254

Kostić, A., \& Chadee, D. (2017). Time Perspective: theory and practice. Londres, Reino Unido. Palgrave Macmillan. https:// 
doi.org/10.1057/978-1-137-60191-9

Laghi, F., Baiocco, R., D’Alessio, M., \& Gurrieri, G. (2009). Suicidal ideation and time perspective in high school students. European Psychiatry, 24, 41-46. https://doi.org/10.1016/ j.eurpsy.2008.08.006

Lewin, K. (1942). Time perspective and morale. En G. Watson (Ed.), Civilian morale: Second yearbook of the Society for the Psychological Study of Social Issued, (pp. 48-70). Houghton Mifflin Company.

Marcial, N., Peña, B. V., Escobedo, J. S., \& Macias, A. (2016). Elementos objetivos y subjetivos en la calidad de vida de hogares rurales en Yehualtepec, Puebla. Estudios sociales. Revista de Alimentación Contemporanea y Desarrollo Regional, 48(26), 279-303. http:// www.scielo.org.mx/pdf/estsoc/v26n48/01884557-estsoc-26-48-00277.pdf

Martínez, P. (2004). Perspectiva temporal futura y satisfacción con la vida a lo largo del ciclo vital [tesis doctoral, Universitat Autonoma de Barcelona]. Tesis Doctorales en Red. https:// www.tdx.cat/handle/10803/4747\#page $=1$

McKay, M. T., Worrell, F. C., Zivkovic, U., Temple, E., Mello, Z. R., \& Musil, B. (2018). A balanced time perspective: Is it an exercise in empiricism, and does it relate mean- ingfully to health and well-being outcomes? International Journal of Psychology. https://doi.org/10.1002/ ijop. 12530.

Mello, Z. R. (2019) A Construct Matures: Time Perspective's multidimensional, developmental, and modifiable qualities. Research in Human Development, 16(2), 93-101. https:// doi.org/10.1080/15427609.2019.1651156

Mello, Z. R., \& Worrell, F. C. (2015). The past, the present, and the future: A conceptual model of time perspective in adolescence. In M. Stolar

ki, N. Fieulaine, \& W. van Beek (Eds.), Time perspective theory; Review, research and application: Essays in honor of Philip G. Zimbardo (pp 115 -129). Springer. https://doi.org/10.1007/978-3319-07368-2_7

Muñoz-Munguía, A. M., Borbón-Morales, C. G., \& Laborín-Álvarez, J. F. (2019). Economía del Comportamiento: un campo fértil para la investigación de aplicaciones en política pública para México. Estudios sociales. Revista de alimentación contemporánea y desarrollo regional, 29(53), e19715. https://doi.org/10.24836/ es.v29i53.715

Nuttin, J., \& Lens, W. (1984). Future time perspective and motivation: theory and research method.
Michigan University. Leuven University Press. Organización para la Cooperación y el Desarrollo Económico. Índice para una vida mejor. Consultado el 28 de agosto 2020. https:// www.oecdbetterlifeindex.org/es/ \#/11111111111

Padawer, E. A., Jacobs-Lawson, J. M., Hershey, D. A., \& Thomas, D. G. (2007). Demographic indicators as predictors of future time perspective. Current Psychology, 26, 102-108. http:// dx.doi.org/10.1007/s12144- 007-9008-4.

Palomar, L. J., \& Lanzagorta, N. (2005). Pobreza, recursos psicologicos y movilidad social. Revista Latinoamericana de Psicología, 37(1),9-45. https://www.redalyc.org/articulo.oa? $\underline{\mathrm{id}=80537101}$

Peng C., Yue C., Avitt A., \& Chen Y. (2021) A Systematic Review Approach to Find Robust Items of the Zimbardo Time Perspective Inventory. Front. Psychol., 12, 627578. https:// doi.org/10.3389/fpsyg.2021.627578

Przepiorka, A., \& Sobol-Kwapinska, M. (2020). People with Positive Time Perspective are More Grateful and Happier: Gratitude Mediates the Relationship Between Time Perspective and Life Satisfaction. Journal of Happiness Studies, https://doi.org/10.1007/s10902-020-00221-z

Seginer, R. (2017). Future orientation and psychological well-being in adolescence: two multiplestep models. En: A. Kostić, \& D. Chadee (Eds), Time Perspective: theory and practice, (pp 339 -365). Palgrave Macmillan. https:// doi.org/10.1057/978-1-137-60191-9_15

Sen, A. (2000). Desarrollo y libertad. Planeta.

Stolarski, M., Bitner, J., \& Zimbardo, P. G. (2011). Time perspective, emotional intelligence and discounting of delayed awards. Time $\&$ Society, 20(3), 346-363.

Stolarski, M., Fieulaine, N., \& Van Beek, W. (2015). Time Perspective Theory; Review, Research and Application. Essays in honor of Phillip Zimbardo. Springer. https://link.springer.com/ book/10.1007/978-3-319-07368-2

Stolarski, M., \& Matthews, G. (2016). Time Perspectives predict mood states and satisfaction with life over and above personality. Current Psychology, 35, 516-526. https://doi.org/10.1007/ s12144-016-9515-2

Stolarski, M., Zajenkowski, M., Jankowski, K. S., \& Szymaniak, K. (2020). Deviation from the balanced time perspective: A systematic review of empirical relationships with psychological variables. Personality and Individual Differences, 156(2020) 109772. https://doi.org/10.1016/ 
j.paid.2019.109772

Stoltman, J. J. K. (2017). Time perspective, health behaviors and well-being in adults [Tesis de maestría inedita, Colegio Eberly de Artes y Ciencias de la Universidad de Virgnia]. Repositorio de Investigación de la Universidad West Virginia. https://researchrepository.wvu.edu/ cgi/viewcontent.cgi?article $=7775 \&$ context $=$ etd

Strathman, A., Gleicher, F., Boninger, D. S., \& Edwards, C. S. (1994). The Consideration of Future Consequences: Weighing Immediate and Distant Outcomes of Behavior. Journal of Personality and Social Psychology, 66(4), 742$752 . \quad$ https://doi.org/10.1037/00223514.66.4.742.

Thompson, C. W., \& Fitzpatrick, J. J. (2007). Positive health practices and temporal perspectives in low income adults. Journal of Clinical Nursing, 17, 1708-1717. https://doi.org/10.1111/j.13652702.2007.02224.x

Thornton, P, (2017). Los grandes economistas. Ciudad de México, México: Crítica.

Trommsdorff, G. (1983). Future orientation and socialization. International Journal of Psychology, 18, 381-406. https://www.researchgate.net/ publica-

tion/30014195_Future_Orientation_and_Social ization

Urzúa, A., \& Caqueo-Urizar, A. (2012). Calidad de vida: Una revisión teórica del concepto. Terapia Psicológica, 30(1), 718-4808. https:// doi.org/10.4067/S0718-48082012000100006.

Urzúa, A., Caqueo-urizar, A., \& Beyle, C. (2018). Procesos cognitivos vinculados al autoreporte de la Calidad de Vida: el efecto de la comparación en población adulta. Interciencia, 43(May), 351 -357. https://www.interciencia.net/wp-content/ uploads/2018/05/351-URZUA-43_5.pdf

Veenhoven, R. (2006). Las cuatro calidades de vida. Organización de conceptos y medidas de la buena vida (Ruiz Ogarrio \& Vargas Juárez, Trad.). Journal of Happiness Studies, 1, 1-39. https:// personal.eur.nl/veenhoven/Pub2000s/2000cfulls.pdf

Worrell, F. (2021). [International Time Perspective Network (13 julio 2021)]. Time Perspective An Historical Developmental Overview [video]. Youtube. https://www.youtube.com/watch? $\mathrm{v}=\mathrm{zsEe} 0 \mathrm{OuB}$ Aw

Zamora Menéndez, Á., Gil Flores, G., \& de Besa Gutierrez, M. R. (2020). Enfoques de aprendizaje y perspectiva temporal: persistencia en estudiantes universitarios. Educación XXI, 23(2), 17-39. https://doi.org/10.5944/educxx1.25552

Zimbardo, P. G., \& Boyd, J. N. (1999). Putting time in perspective: A valid, reliable individualdifferences metric. Journal of Personality and Social Psychology, 77, 1271-1288. https:// doi.org/10.1037/0022-3514.77.6.1271

Zimbardo, P. G., \& Boyd, J. N. (2009). La Paradoja del Tiempo. La Nueva Psicología del Tiempo. Editorial Paidós.

Zimbardo P. G., \& Boyd J. N. (2015) Putting Time in Perspective: A Valid, Reliable IndividualDifferences Metric. En: Stolarski M., Fieulaine N., van Beek W. (Eds) Time Perspective Theory; Review, Research and Application (pp 1756). Cham, Suiza, Springer. https:// doi.org/10.1007/978-3-319-07368-2_2

Zimbardo P. G., Clements N., \& Rego Leite U. (2017). Time Perspective and Financial Health: To Improve Financial Health, Traditional Financial Literacy Skills Are Not Sufficient. Understanding Your Time Perspective Is Critical. En Kostić, A., Chadee, D. (Eds), Time Perspective: theory and practice. (pp. 9-41). Palgrave Macmillan. https://link.springer.com/ chapter/10.1057/978-1-137-60191-9_2

Zimbardo, P. G., Sword, R., \& Sword, R. (2012). The Time Cure: Overcoming PTSD with the New Psychology of Time Perspective Therapy. https://www.wiley.com/enmx/ The+Time+Cure:+Overcoming + PTSD + with $+\mathrm{t}$ he + New + Psychology + of + Time + Perspective $+\mathrm{T}$ herapy-p-9781118205679 